\title{
Characterization of variable Besov-type spaces by ball means of differences
}

\author{
Douadi Drihem
}

\begin{abstract}
With the help of the maximal function characterizations of Besov-type spaces with variable smoothness and integrability we prove the characterization by ball means of differences for these function spaces.
\end{abstract}

\section{Introduction}

Function spaces with variable exponents have been intensively studied in recent years by a significant number of authors. The motivation for the increasing interest in such spaces comes not only from theoretical purposes, but also from applications to fluid dynamics [26], image restoration [2], and PDEs with nonstandard growth conditions. A comprehensive overview on existence and regularity results for PDEs in the variable exponent setting, including an extensive list of references on this subject, is given in the recent survey [15]. In all these applications the Lebesgue and Sobolev spaces with variable integrability, $L^{p(\cdot)}$ and $W^{k, p(\cdot)}$, seem to appear in a natural way.

Lebesgue spaces with variable exponent have been explicitly studied in [27], but the systematic study of the spaces $L^{p(\cdot)}$ and $W^{k, p(\cdot)}$ started in [18]. Since then, various other function spaces and classical operators of harmonic analysis have been investigated in the variable exponent setting, notably after the boundedness of the Hardy-Littlewood maximal operator was proved in [4]. We only refer to the survey monograph [7] for further details and references on recent developments in this field.

In recent years, there has been growing interest in generalizing classical spaces such as Sobolev spaces, Besov spaces, and Triebel-Lizorkin spaces to the case with either variable integrability or variable smoothness (see Triebel's monographs [34] and [35] for the history of these function spaces).

Besov spaces of variable smoothness and integrability, $B_{p(\cdot), q(\cdot)}^{\alpha(\cdot)}$, initially appeared in the paper of A. Almeida and P. Hästö [1]. Several basic properties were established, such as the Fourier analytical characterization. When $p, q, \alpha$ are constants they coincide with the usual function spaces $B_{p, q}^{\alpha}$. Also Sobolev-type embeddings and the characterization by approximations of these function spaces

Kyoto Journal of Mathematics, Vol. 56, No. 3 (2016), 655-680

DOI 10.1215/21562261-3600220, (C) 2016 by Kyoto University

Received March 27, 2015. Revised April 13, 2015. Accepted April 16, 2015.

2010 Mathematics Subject Classification: Primary 46E35; Secondary 42B25, 42B35. 
were obtained. Taking $\alpha \in \mathbb{R}$ and $q \in(0, \infty]$ as constants we derive the spaces $B_{p(\cdot), q}^{\alpha}$ studied by $\mathrm{Xu}$ [37], [38]. Some properties of these function spaces such as local means characterizations, atomic decomposition, and characterizations by ball means of differences can be found in [9] and [16]. Variable Besov-type spaces have been introduced in [13] and [12], where their basic properties are given, such as the Sobolev-type embeddings and that under some conditions these spaces are just the Besov spaces $B_{\infty, \infty}^{\alpha(\cdot)+n(1 / \tau(\cdot)-1 / p(\cdot))}$. For constant exponents, these spaces unify and generalize many classical function spaces including Besov spaces and Besov-Morrey spaces (see, e.g., [43, Corollary 3.3]).

The main aim of this article is to prove the characterization by ball means of differences for Besov-type spaces with variable smoothness and integrability.

\section{Preliminaries}

As usual, we denote by $\mathbb{R}^{n}$ the $n$-dimensional real Euclidean space, $\mathbb{N}$ the collection of all natural numbers, and $\mathbb{N}_{0}=\mathbb{N} \cup\{0\}$. The letter $\mathbb{Z}$ stands for the set of all integer numbers. For a multi-index $\alpha=\left(\alpha_{1}, \ldots, \alpha_{n}\right) \in \mathbb{N}_{0}^{n}$, we write $|\alpha|=\alpha_{1}+\cdots+\alpha_{n}$. The Euclidean scalar product of $x=\left(x_{1}, \ldots, x_{n}\right)$ and $y=$ $\left(y_{1}, \ldots, y_{n}\right)$ is given by $x \cdot y=x_{1} y_{1}+\cdots+x_{n} y_{n}$.

For $x \in \mathbb{R}^{n}$ and $r>0$ we denote by $B(x, r)$ the open ball in $\mathbb{R}^{n}$ with center $x$ and radius $r$. By supp $f$ we denote the support of the function $f$, that is, the closure of its nonzero set. If $E \subset \mathbb{R}^{n}$ is a measurable set, then $|E|$ stands for the (Lebesgue) measure of $E$ and $\chi_{E}$ denotes its characteristic function.

The symbol $\mathcal{S}\left(\mathbb{R}^{n}\right)$ is used in place of the set of all Schwartz functions $\varphi$ on $\mathbb{R}^{n}$, that is, $\varphi$ is infinitely differentiable and

$$
\|\varphi\|_{k, l}:=\sup _{x \in \mathbb{R}^{n}} \sum_{|\alpha| \leq l}\left|D^{\alpha} \varphi(x)\right|(1+|x|)^{k}<\infty
$$

for all $k, l \in \mathbb{N}$. We denote by $\mathcal{S}^{\prime}\left(\mathbb{R}^{n}\right)$ the dual space of all tempered distributions on $\mathbb{R}^{n}$. We define the Fourier transform of a function $f \in \mathcal{S}\left(\mathbb{R}^{n}\right)$ by

$$
\mathcal{F}(f)(\xi)=(2 \pi)^{-n / 2} \int_{\mathbb{R}^{n}} e^{-i x \cdot \xi} f(x) d x .
$$

Its inverse is denoted by $\mathcal{F}^{-1} f$. Both $\mathcal{F}$ and $\mathcal{F}^{-1}$ are extended to the dual Schwartz space $\mathcal{S}^{\prime}\left(\mathbb{R}^{n}\right)$ in the usual way.

The Hardy-Littlewood maximal operator $\mathcal{M}$ is defined on $L_{\text {loc }}^{1}$ by

$$
\mathcal{M} f(x)=\sup _{r>0} \frac{1}{|B(x, r)|} \int_{B(x, r)}|f(y)| d y .
$$

For $v \in \mathbb{Z}$ and $m=\left(m_{1}, \ldots, m_{n}\right) \in \mathbb{Z}^{n}$, let $Q_{v, m}$ be the dyadic cube in $\mathbb{R}^{n}, Q_{v, m}=$ $\left\{\left(x_{1}, \ldots, x_{n}\right): m_{i} \leq 2^{v} x_{i}<m_{i}+1, i=1,2, \ldots, n\right\}$. For the collection of all such cubes we use $\mathcal{Q}=\left\{Q_{v, m}: v \in \mathbb{Z}, m \in \mathbb{Z}^{n}\right\}$. For each cube $Q$, we denote by $x_{Q_{v, m}}$ the lower left corner $2^{-v} m$ of $Q=Q_{v, m}$ and its side length by $l(Q)$. Furthermore, we put $v_{Q}=-\log _{2} l(Q)$ and $v_{Q}^{+}=\max \left(v_{Q}, 0\right)$.

By $C$ we denote generic positive constants, which may have different values at different occurrences. Although the exact values of the constants are usually 
irrelevant for our purposes, sometimes we emphasize their dependence on certain parameters (e.g., $C(p)$ means that $C$ depends on $p$ ). Further notation will be properly introduced whenever needed.

The variable exponents that we consider are always measurable functions $p$ on $\mathbb{R}^{n}$ with range in $[C, \infty[$ for some $C>0$. We denote the set of such functions by $\mathcal{P}_{0}$. The subset of variable exponents with range $[1, \infty[$ is denoted by $\mathcal{P}$. We use the standard notation

$$
p^{-}=\underset{x \in \mathbb{R}^{n}}{\operatorname{ess-inf}} p(x), \quad p^{+}=\underset{x \in \mathbb{R}^{n}}{\operatorname{ess-sup}} p(x) .
$$

The variable exponent modular is defined by $\varrho_{p(\cdot)}(f)=\int_{\mathbb{R}^{n}} \varrho_{p(x)}(|f(x)|) d x$, where $\varrho_{p}(t)=t^{p}$. The variable exponent Lebesgue space $L^{p(\cdot)}$ consists of measurable functions $f$ on $\mathbb{R}^{n}$ such that $\varrho_{p(\cdot)}(\lambda f)<\infty$ for some $\lambda>0$. We define the Luxemburg (quasi-)norm on this space by the formula $\|f\|_{p(\cdot)}=\inf \{\lambda>0$ : $\left.\varrho_{p(\cdot)}\left(\frac{f}{\lambda}\right) \leq 1\right\}$. A useful property is that $\|f\|_{p(\cdot)} \leq 1$ if and only if $\varrho_{p(\cdot)}(f) \leq 1$ (see [7, Lemma 3.2.4]).

Let $p, q \in \mathcal{P}_{0}$. The mixed Lebesgue-sequence space $\ell^{q(\cdot)}\left(L^{p(\cdot)}\right)$ is defined on sequences of $L^{p(\cdot)}$-functions by the modular

$$
\varrho_{\ell^{q(\cdot)}\left(L^{p(\cdot)}\right)}\left(\left(f_{v}\right)_{v}\right):=\sum_{v} \inf \left\{\lambda_{v}>0: \varrho_{p(\cdot)}\left(\frac{f_{v}}{\lambda_{v}^{1 / q(\cdot)}}\right) \leq 1\right\} .
$$

The (quasi-)norm is defined from this as usual:

$$
\left\|\left(f_{v}\right)_{v}\right\|_{\ell^{q(\cdot)}\left(L^{p(\cdot)}\right)}:=\inf \left\{\mu>0: \varrho_{\ell^{q(\cdot)}\left(L^{p(\cdot)}\right)}\left(\frac{1}{\mu}\left(f_{v}\right)_{v}\right) \leq 1\right\} .
$$

If $q^{+}<\infty$, then we can replace (2.1) by the simpler expression $\varrho_{\ell^{q(\cdot)}\left(L^{p(\cdot)}\right)}\left(\left(f_{v}\right)_{v}\right)=$ $\sum_{v}\left\|\left|f_{v}\right|^{q(\cdot)}\right\|_{\frac{p(\cdot)}{q(\cdot)}}$. Furthermore, if $p$ and $q$ are constants, then $\ell^{q(\cdot)}\left(L^{p(\cdot)}\right)=$ $\ell^{q}\left(L^{p}\right)$. The case $p \equiv \infty$ can be included by replacing the last modular by $\varrho_{\ell^{q(\cdot)}\left(L^{\infty}\right)}\left(\left(f_{v}\right)_{v}\right)=\sum_{v}\left|\left\|\left|f_{v}\right|^{q(\cdot)}\right\|_{\infty}\right.$.

It is known (cf. [1] and [17]) that $\ell^{q(\cdot)}\left(L^{p(\cdot)}\right)$ is a norm if $q(\cdot) \geq 1$ is constant almost everywhere (a.e.) on $\mathbb{R}^{n}$ and $p(\cdot) \geq 1$, or if $\frac{1}{p(x)}+\frac{1}{q(x)} \leq 1$ a.e. on $\mathbb{R}^{n}$, or if $1 \leq q(x) \leq p(x)<\infty$ a.e. on $\mathbb{R}^{n}$.

We say that $g: \mathbb{R}^{n} \rightarrow \mathbb{R}$ is locally log-Hölder continuous, abbreviated $g \in$ $C_{\text {loc }}^{\text {log }}$, if there exists $c_{\log }(g)>0$ such that

$$
|g(x)-g(y)| \leq \frac{c_{\log }(g)}{\log (e+1 /|x-y|)}
$$

for all $x, y \in \mathbb{R}^{n}$. We say that $g$ satisfies the log-Hölder decay condition if there exists $g_{\infty} \in \mathbb{R}$ and a constant $c_{\log }>0$ such that

$$
\left|g(x)-g_{\infty}\right| \leq \frac{c_{\log }}{\log (e+|x|)}
$$

for all $x \in \mathbb{R}^{n}$. We say that $g$ is globally log-Hölder continuous, abbreviated $g \in C^{\log }$, if it is locally log-Hölder continuous and satisfies the log-Hölder decay condition. The constants $c_{\log }(g)$ and $c_{\log }$ are called the locally log-Hölder constant and the log-Hölder decay constant, respectively. We note that all functions $g \in C_{\mathrm{loc}}^{\log }$ always belong to $L^{\infty}$. 
We define the following class of variable exponents:

$$
\mathcal{P}^{\log }:=\left\{p \in \mathcal{P}: \frac{1}{p} \text { is globally log-Hölder continuous }\right\},
$$

which were introduced in $\left[6\right.$, Section 2]. We define $1 / p_{\infty}:=\lim _{|x| \rightarrow \infty} 1 / p(x)$ and we use the convention $\frac{1}{\infty}=0$. Note that, although $\frac{1}{p}$ is bounded, the variable exponent $p$ itself can be unbounded. It was shown in [7, Theorem 4.3.8] that $\mathcal{M}: L^{p(\cdot)} \rightarrow L^{p(\cdot)}$ is bounded if $p \in \mathcal{P}^{\log }$ and $p^{-}>1$ (see also [6, Theorem 1.2]). Also if $p \in \mathcal{P}^{\log }$, then the convolution with a radially decreasing $L^{1}$-function is bounded on $L^{p(\cdot)}:\|\varphi * f\|_{p(\cdot)} \leq C\|\varphi\|_{1}\|f\|_{p(\cdot)}$. We also refer to the papers [3] and [4], where various results on maximal function in variable Lebesgue spaces were obtained.

Very often we have to deal with the norm of characteristic functions on balls (or cubes) when studying the behavior of various operators in harmonic analysis. In classical $L^{p}$-spaces the norm of such functions is easily calculated, but this is not the case when we consider variable exponents. Nevertheless, it is known that for $p \in \mathcal{P}^{\log }$ we have

$$
\left\|\chi_{B}\right\|_{p(\cdot)}\left\|\chi_{B}\right\|_{p^{\prime}(\cdot)} \approx|B|
$$

Also,

$$
\left\|\chi_{B}\right\|_{p(\cdot)} \approx|B|^{\frac{1}{p(x)}}, \quad x \in B
$$

for small balls $B \subset \mathbb{R}^{n}\left(|B| \leq 2^{n}\right)$, and

$$
\left\|\chi_{B}\right\|_{p(\cdot)} \approx|B|^{\frac{1}{p_{\infty}}}
$$

for large balls $(|B| \geq 1)$, with constants only depending on the log-Hölder constant of $p$ (see, for example, [7, Section 4.5]), where $f \approx g$ means $C_{1} f \leq g \leq C_{2} f$.

Recall that $\eta_{v, m}(x)=2^{n v}\left(1+2^{v}|x|\right)^{-m}$, for any $x \in \mathbb{R}^{n}, v \in \mathbb{N}_{0}$, and $m>0$. Note that $\eta_{v, m} \in L^{1}$ when $m>n$ and that $\left\|\eta_{v, m}\right\|_{1}=C_{m}$ is independent of $v$.

\subsection{Some technical lemmas}

In this section we present some results which are useful for us. The following lemma is from [16, Lemma 19] (see also [5, Lemma 6.1]).

\section{LEMMA 2.5}

Let $\alpha \in C_{\operatorname{loc}}^{\log }$, and let $R \geq c_{\log }(\alpha)$, where $c_{\log }(\alpha)$ is the constant from (2.2) for $\alpha$. Then

$$
2^{v \alpha(x)} \eta_{v, m+R}(x-y) \leq C 2^{v \alpha(y)} \eta_{v, m}(x-y)
$$

with $C>0$ independent of $x, y \in \mathbb{R}^{n}$ and $v, m \in \mathbb{N}_{0}$.

The previous lemma allows us to treat the variable smoothness in many cases as if it were not variable at all; namely, we can move the term inside the convolution as follows:

$$
2^{v \alpha(x)} \eta_{v, m+R} * f(x) \leq C \eta_{v, m} *\left(2^{v \alpha(\cdot)} f\right)(x) .
$$


LEMMA 2.6

Let $r, R, N>0$, let $m>n$, and let $\theta, \omega \in \mathcal{S}\left(\mathbb{R}^{n}\right)$ with $\operatorname{supp} \mathcal{F} \omega \subset B(0,1)$. Then there exists $C=C(r, m, n)>0$ such that, for all $g \in \mathcal{S}^{\prime}\left(\mathbb{R}^{n}\right)$, we have

$$
\left|\theta_{R} * \omega_{N} * g(x)\right| \leq C \max \left(1,\left(\frac{N}{R}\right)^{m}\right)\left(\eta_{N, m} *\left|\omega_{N} * g\right|^{r}(x)\right)^{1 / r}, \quad x \in \mathbb{R}^{n},
$$

where $\theta_{R}(\cdot)=R^{n} \theta(R \cdot), \omega_{N}(\cdot)=N^{n} \omega(N \cdot)$, and $\eta_{N, m}(\cdot)=N^{n}(1+N|\cdot|)^{-m}$.

This lemma is a slight variant of [33, Chapter V, Theorem 5] (see also [5, Lemma A.7], [12, Lemma 2.2]). The following lemma is from [13, Lemma 2.11].

\section{LEMMA 2.7}

Let $\tau \in \mathcal{P}_{0}^{\log }$ and $k \in \mathbb{Z}^{n}$. For any cubes $P$ and $Q$ such that $P \subset Q$, we have

$$
C_{1}\left(\frac{|Q|}{|P|}\right)^{1 / \tau^{+}} \leq \frac{\left\|\chi_{Q}\right\|_{\tau(\cdot)}}{\left\|\chi_{P}\right\|_{\tau(\cdot)}} \leq C_{2}\left(\frac{|Q|}{|P|}\right)^{1 / \tau^{-}},
$$

where $C_{1}, C_{2}>0$ are independent of $|Q|$ and $|P|$.

Let $L_{\tau(\cdot)}^{p(\cdot)}$ be the collection of functions $f \in L_{\text {loc }}^{p(\cdot)}\left(\mathbb{R}^{n}\right)$ such that

$$
\|f\|_{L_{\tau(\cdot)}^{p(\cdot)}}:=\sup \left\|\frac{f \chi_{P}}{\left\|\chi_{P}\right\|_{\tau(\cdot)}}\right\|_{p(\cdot)}<\infty, \quad p, \tau \in \mathcal{P}_{0},
$$

where the supremum is taken over all dyadic cubes $P$ with $|P| \geq 1$. Also, the space $\widetilde{L^{p(\cdot)}}$ is defined to be the set of all functions $f$ such that

$$
\|f\|_{\widetilde{L^{p(\cdot)}}}:=\sup \left\|f \chi_{P}\right\|_{p(\cdot)}<\infty, \quad p \in \mathcal{P}_{0},
$$

where the supremum is taken over all dyadic cubes $P$ with $|P|=1$. Obviously $L_{\tau(\cdot)}^{p(\cdot)} \hookrightarrow \widetilde{L^{p(\cdot)}}$. We introduce the abbreviations

$$
\begin{aligned}
&\left\|\left(f_{v}\right)_{v}\right\|_{\ell^{q(\cdot)}\left(L_{p(\cdot)}^{p(\cdot)}\right)}:=\sup _{\{P \in \mathcal{Q},|P| \leq 1\}}\left\|\left(\frac{f_{v}}{|P|^{1 / p(\cdot)}} \chi_{P}\right)_{v \geq v_{P}}\right\|_{\ell^{q(\cdot)}\left(L^{p(\cdot)}\right)}, \\
&\left\|\left(f_{v}\right)_{v}\right\|_{\ell^{\tau(\cdot), q(\cdot)\left(L^{p(\cdot)}\right)}}:=\sup _{P \in \mathcal{Q}}\left\|\left(\frac{f_{v}}{\left\|\chi_{P}\right\|_{\tau(\cdot)}} \chi_{P}\right)_{v \geq v_{P}^{+}}\right\|_{\ell^{q(\cdot)}\left(L^{p(\cdot)}\right)} \cdot
\end{aligned}
$$

The following lemma is the $\ell^{q(\cdot)}\left(L_{p(\cdot)}^{p(\cdot)}\right)\left(-\ell^{\tau(\cdot), q(\cdot)}\left(L^{p(\cdot)}\right)\right)$-version of Lemma 4.7 from A. Almeida and P. Hästö [1]. (We use it, since the maximal operator is in general not bounded on $\ell^{q(\cdot)}\left(L^{p(\cdot)}\right)$ (see [1, Example 4.1])).

\section{LEMMA 2.8}

Let $p \in \mathcal{P}^{\log }$ and $q, \tau \in \mathcal{P}_{0}^{\log }$ with $0<q^{-} \leq q^{+}<\infty$.

(i) For $m>2 n+c_{\log }(1 / \tau)+c_{\log }(1 / q)$, we have

$$
\left\|\left(\eta_{v, m} * f_{v}\right)_{v}\right\|_{\ell^{\tau(\cdot), q(\cdot)}\left(L^{p(\cdot)}\right)} \leq C\left\|\left(f_{v}\right)_{v}\right\|_{\ell^{\tau(\cdot), q(\cdot)}\left(L^{p(\cdot)}\right)},
$$

where $C>0$ is independent of $\left\{f_{v}\right\}_{v \in \mathbb{N}_{0}}$. 
(ii) For $m>2 n+c_{\log }(1 / p)+c_{\log }(1 / q)$, we have

$$
\left\|\left(\eta_{v, m} * f_{v}\right)_{v}\right\|_{\ell^{q(\cdot)}\left(L_{p(\cdot)}^{p(\cdot)}\right)} \leq C\left\|\left(f_{v}\right)_{v}\right\|_{\ell^{q(\cdot)}\left(L_{p(\cdot)}^{p(\cdot)}\right)},
$$

where $C>0$ is independent of $\left\{f_{v}\right\}_{v \in \mathbb{N}_{0}}$.

The proof is given in [13, Lemma 2.12]. The next three lemmas are from [5] where the first tells us that in most circumstances two convolutions are as good as one.

\section{LEMMA 2.9}

For $v_{0}, v_{1} \in \mathbb{N}_{0}$ and $m>n$, we have

$$
C_{2} \eta_{\min \left(v_{0}, v_{1}\right), m} \leq \eta_{v_{0}, m} * \eta_{v_{1}, m} \leq C_{1} \eta_{\min \left(v_{0}, v_{1}\right), m},
$$

where $C_{1}, C_{2}>0$ depend only on $m$ and $n$.

\section{LEMMA 2.10}

Let $\alpha \in C_{\operatorname{loc}}^{\log }$ and $p, q, \tau \in \mathcal{P}_{0}^{\log }$ with $0<q^{-} \leq q^{+}<\infty$. Let $\left\{f_{k}\right\}_{k \in \mathbb{N}_{0}}$ be a sequence of measurable functions on $\mathbb{R}^{n}$. For all $v \in \mathbb{N}_{0}, P \in \mathcal{Q}$, and $x \in \mathbb{R}^{n}$, let $g_{v}(x)=$ $\sum_{k=v_{P}^{+}}^{v} 2^{(k-v) \delta} f_{k}(x)$. Then there exists a positive constant $C$ that is independent of $\left\{f_{k}\right\}_{k \in \mathbb{N}_{0}}$ such that

$$
\left\|\left(\frac{g_{v}}{\left\|\chi_{P}\right\|_{\tau(\cdot)}} \chi_{P}\right)_{v \geq v_{P}^{+}}\right\|_{\ell^{q(\cdot)}\left(L^{p(\cdot)}\right)} \leq C\left\|\left(\frac{f_{v}}{\left\|\chi_{P}\right\|_{\tau(\cdot)}} \chi_{P}\right)_{v \geq v_{P}^{+}}\right\|_{\ell^{q(\cdot)}\left(L^{p(\cdot)}\right)}
$$

for any dyadic cube $P$ and

$$
\left\|\left(\frac{g_{v}}{|P|^{1 / p(\cdot)}} \chi_{P}\right)_{v \geq v_{P}}\right\|_{\ell^{q(\cdot)}\left(L^{p(\cdot)}\right)} \leq C\left\|\left(\frac{f_{v}}{|P|^{1 / p(\cdot)}} \chi_{P}\right)_{v \geq v_{P}}\right\|_{\ell^{q(\cdot)}\left(L^{p(\cdot)}\right)}
$$

for any dyadic cube $P$ with $|P| \leq 1$.

The proof of Lemma 2.10 can be found in [12].

\section{Variable Besov-type spaces}

In this section we present the Fourier analytical definition of Besov-type spaces of variable smoothness and integrability, and we recall their basic properties. Let $\Psi$ be a function in $\mathcal{S}\left(\mathbb{R}^{n}\right)$ satisfying $0 \leq \Psi(x) \leq 1$ for all $x, \Psi(x)=1$ for $|x| \leq 1$, and $\Psi(x)=0$ for $|x| \geq 2$. We put $\mathcal{F} \varphi_{0}(x)=\Psi(x), \mathcal{F} \varphi(x)=\Psi\left(\frac{x}{2}\right)-\Psi(x)$, and $\mathcal{F} \varphi_{v}(x)=\mathcal{F} \varphi\left(2^{-v+1} x\right)$ for $v=1,2,3, \ldots$ Then $\left\{\mathcal{F} \varphi_{v}\right\}_{v \in \mathbb{N}_{0}}$ is a resolution of unity, $\sum_{v=0}^{\infty} \mathcal{F} \varphi_{v}(x)=1$ for all $x \in \mathbb{R}^{n}$. Thus, we obtain the Littlewood-Paley decomposition

$$
f=\sum_{v=0}^{\infty} \varphi_{v} * f
$$

of all $f \in \mathcal{S}^{\prime}\left(\mathbb{R}^{n}\right)$ (convergence in $\mathcal{S}^{\prime}\left(\mathbb{R}^{n}\right)$ ).

Now, we define the spaces under consideration. 


\section{DEFINITION 3.2}

Let $\left\{\mathcal{F} \varphi_{v}\right\}_{v \in \mathbb{N}_{0}}$ be a resolution of unity, let $\alpha: \mathbb{R}^{n} \rightarrow \mathbb{R}$, and let $p, q, \tau \in \mathcal{P}_{0}$. that

(i) The Besov-type space $\widetilde{B}_{p(\cdot), q(\cdot)}^{\alpha(\cdot), p(\cdot)}$ is the collection of all $f \in \mathcal{S}^{\prime}\left(\mathbb{R}^{n}\right)$ such

$$
\|f\|_{\widetilde{B}_{p(\cdot), q(\cdot)}^{\alpha(\cdot), p(\cdot)}}:=\sup _{P \in \mathcal{Q}}\left\|\left(\frac{2^{v \alpha(\cdot)} \varphi_{v} * f}{|P|^{1 / p(\cdot)}} \chi_{P}\right)_{v \geq v_{P}^{+}}\right\|_{\ell^{q(\cdot)\left(L^{p(\cdot)}\right)}}<\infty .
$$
that

(ii) The Besov-type space $B_{p(\cdot), q(\cdot)}^{\alpha(\cdot), \tau(\cdot)}$ is the collection of all $f \in \mathcal{S}^{\prime}\left(\mathbb{R}^{n}\right)$ such

$$
\|f\|_{B_{p(\cdot), q(\cdot)}^{\alpha(\cdot), \tau(\cdot)}}:=\sup _{P \in \mathcal{Q}}\left\|\left(\frac{2^{v \alpha(\cdot)} \varphi_{v} * f}{\left\|\chi_{P}\right\|_{\tau(\cdot)}} \chi_{P}\right)_{v \geq v_{P}^{+}}\right\|_{\ell^{q(\cdot)}\left(L^{p(\cdot)}\right)}<\infty .
$$

Since $\mathcal{F} \varphi_{v}$ is smooth, $\mathcal{F} \varphi_{v} \cdot \mathcal{F} f$ makes sense as a distribution in $\mathcal{S}^{\prime}\left(\mathbb{R}^{n}\right)$. Because of the compactness of the support of $\mathcal{F} \varphi_{v}$ the famous Paley-Wiener-Schwartz theorem (see [34, Theorem 1.2.1]) tells us that $\varphi_{v} * f$ is an entire analytic function. So the quasinorms in (3.3) and (3.4) make sense.

The definitions of the spaces $\widetilde{B}_{p(\cdot), q(\cdot)}^{\alpha(\cdot), p(\cdot)}$ and $B_{p(\cdot), q(\cdot)}^{\alpha(\cdot),(\cdot)}$ are independent of the chosen resolution of unity (3.1) if $\alpha \in C_{\operatorname{loc}}^{\log }, p, q, \tau \in \mathcal{P}_{0}^{\log }$, and $0<q^{+}<\infty$, and different choices yield equivalent quasinorms. Using the system $\left\{\mathcal{F} \varphi_{v}\right\}_{v \in \mathbb{N}_{0}}$ we can define the norm

$$
\|f\|_{B_{p, q}^{\alpha, \tau}}:=\sup _{P \in \mathcal{Q}} \frac{1}{|P|^{\tau}}\left(\sum_{v=v_{P}^{+}}^{\infty} 2^{v \alpha q}\left\|\left(\varphi_{v} * f\right) \chi_{P}\right\|_{p}^{q}\right)^{1 / q}
$$

for constants $\alpha$ and $p, q \in(0, \infty]$. The Besov-type space $B_{p, q}^{\alpha, \tau}$ consists of all distributions $f \in \mathcal{S}^{\prime}\left(\mathbb{R}^{n}\right)$ for which $\|f\|_{B_{p, q}^{\alpha, \tau}}<\infty$. It is well known that these spaces do not depend on the choice of the system $\left\{\mathcal{F} \varphi_{v}\right\}_{v \in \mathbb{N}_{0}}$ (up to equivalence of quasinorms). If $\tau=0$, then $B_{p, q}^{\alpha, 0}=B_{p, q}^{\alpha}$. Further details on the classical theory of these spaces can be found in [8], [10], [20], and [43] (see also [11] for recent developments). Variable Besov-type spaces $\widetilde{B}_{p(\cdot), q(\cdot)}^{\alpha(\cdot), p(\cdot)}$ and $B_{p(\cdot), q(\cdot)}^{\alpha(\cdot), \tau(\cdot)}$ have been introduced in [13] and [12], where their basic properties are given, such as the Sobolev-type embeddings. One recognizes immediately that if $\alpha, \tau, p$, and $q$ are constants, then $\widetilde{B}_{p(\cdot), q(\cdot)}^{\alpha(\cdot)}=B_{p, q}^{\alpha, 1 / p}$ and $B_{p(\cdot), q(\cdot)}^{\alpha(\cdot), \tau(\cdot)}=B_{p, q}^{\alpha, \tau}$. Besov-type space $B_{p(\cdot), \infty}^{\alpha(\cdot), \tau(\cdot)}$ consist of all distributions $f \in \mathcal{S}^{\prime}\left(\mathbb{R}^{n}\right)$ such that

$$
\sup _{P \in \mathcal{Q}, v \geq v_{P}^{+}}\left\|\frac{2^{v \alpha(\cdot)} \varphi_{v} * f}{\left\|\chi_{P}\right\|_{\tau(\cdot)}} \chi_{P}\right\|_{p(\cdot)}<\infty .
$$

Also, we have

$$
2^{v(\alpha(x)+n(1 / \tau(x)-1 / p(x)))}\left|\varphi_{v} * f(x)\right| \leq C\|f\|_{B_{p(\cdot), q(\cdot)}^{\alpha(\cdot),(\cdot)}}
$$

and

$$
2^{v \alpha(x)}\left|\varphi_{v} * f(x)\right| \leq C\|f\|_{\widetilde{B}_{p(\cdot), q(\cdot)}^{\alpha(\cdot),(\cdot)}}
$$


for any $x \in \mathbb{R}^{n}, \alpha \in C_{\mathrm{loc}}^{\mathrm{log}}$, and $p, q, \tau \in \mathcal{P}_{0}^{\log }$, where $C>0$ is independent of $x$ and $v$. In the definition of the spaces $B_{p(\cdot), q(\cdot)}^{\alpha(\cdot)}$ and $\widetilde{B}_{p(\cdot), q(\cdot)}^{\alpha(\cdot),(\cdot)}$ if we replace dyadic cubes $P$ by arbitrary cubes $P$, then we obtain equivalent quasinorms.

Independently, D. Yang, C. Zhuo, and W. Yuan [42] studied the function spaces $B_{p(\cdot), q(\cdot)}^{\alpha(\cdot),(\cdot)}$ where several properties are obtained such as atomic decomposition and the boundedness of the trace operator. The following results are given in $[13$, Theorems 3.8, 4.7] where these results with fixed exponents are proved in [41, Theorem 1] and [43, Proposition 2.6].

\section{THEOREM 3.6}

Let $\alpha \in C_{\mathrm{loc}}^{\mathrm{log}}, p, p_{1}, p_{2}, q, \tau \in \mathcal{P}_{0}^{\log }$, and $0<q^{+}<\infty$.

(i) Let $\tau_{\infty} \in\left(0, p^{-}\right]$. If $(1 / \tau-1 / p)^{-}>0$ or $(1 / \tau-1 / p)^{-} \geq 0$ and $q \equiv \infty$, then

$$
B_{p(\cdot), q(\cdot)}^{\alpha(\cdot), \tau(\cdot)}=B_{\infty, \infty}^{\alpha(\cdot)+n(1 / \tau(\cdot)-1 / p(\cdot))}
$$

with equivalent norms.

(ii) If $\left(p_{2}-p_{1}\right)^{+} \leq 0$, then

$$
B_{p_{2}(\cdot), q(\cdot)}^{\alpha(\cdot)+n / \tau(\cdot)+n / p_{2}(\cdot)-n / p_{1}(\cdot)} \hookrightarrow B_{p_{1}(\cdot), q(\cdot)}^{\alpha(\cdot)}
$$

(iii) We have

$$
B_{p(\cdot), q(\cdot)}^{\alpha(\cdot), \tau(\cdot)} \hookrightarrow B_{\infty, \infty}^{\alpha(\cdot)+n / \tau(\cdot)-n / p(\cdot)}
$$

Here $B_{p(\cdot), q(\cdot)}^{\alpha(\cdot)}$ is the Besov space of variable smoothness and integrability, and it is the collection of all $f \in \mathcal{S}^{\prime}\left(\mathbb{R}^{n}\right)$ such that

$$
\|f\|_{B_{p(\cdot), q(\cdot)}^{\alpha(\cdot)}}:=\left\|\left(2^{v \alpha(\cdot)} \varphi_{v} * f\right)_{v \geq 0}\right\|_{\ell q(\cdot)\left(L^{p(\cdot)}\right)}<\infty,
$$

which was introduced and investigated in [1] (see [9] and [16] for further results). We refer the reader to the recent paper [45] for further details, historical remarks, and more references on embeddings of Besov-type spaces with fixed exponents.

Let $0<u \leq p<\infty$. The Morrey space $\mathcal{M}_{u}^{p}$ is defined to be the set of all $u$-locally Lebesgue-integrable functions $f$ on $\mathbb{R}^{n}$ such that

$$
\|f\|_{\mathcal{M}_{u}^{p}}:=\sup _{B}|B|^{\frac{1}{p}-\frac{1}{u}}\left(\int_{B}|f(x)|^{u} d x\right)^{1 / u}<\infty
$$

where the supremum is taken over all balls $B$ in $\mathbb{R}^{n}$. The spaces $\mathcal{M}_{u}^{p}$ are quasiBanach spaces (Banach spaces for $u \geq 1$ ). They were introduced by Morrey in [22] and belong to the wider class of Morrey-Campanato spaces (cf. [25]). They can be considered as a complement to $L^{p}$-spaces. As a matter of fact, $\mathcal{M}_{p}^{p}=L^{p}$. One can easily see that

$$
\mathcal{M}_{w}^{p} \hookrightarrow \mathcal{M}_{u}^{p} \quad \text { if } 0<u \leq w<\infty .
$$




\section{DEFINITION 3.7}

Let $\left\{\mathcal{F} \varphi_{v}\right\}_{v \in \mathbb{N}_{0}}$ be a resolution of unity, $\alpha: \mathbb{R}^{n} \rightarrow \mathbb{R}, 0<u \leq p<\infty$, and $0<q \leq \infty$. The Besov-Morrey space $\mathcal{N}_{p, q, u}^{\alpha(\cdot)}$ is the collection of all $f \in \mathcal{S}^{\prime}\left(\mathbb{R}^{n}\right)$ such that

$$
\|f\|_{\mathcal{N}_{p, q, u}^{\alpha(\cdot)}}:=\left(\sum_{v=0}^{\infty}\left\|2^{v \alpha(\cdot)} \varphi_{v} * f\right\|_{\mathcal{M}_{u}^{p}}^{q}\right)^{1 / q}<\infty .
$$

Besov-Morrey spaces with fixed exponents were introduced by Netrusov [23]. Kozono and Yamazaki [19] studied semilinear heat equations and Navier-Stokes equations with initial data belonging to Besov-Morrey spaces. The investigations were continued by Mazzucato [21], where one can find the wavelet decomposition of Besov-Morrey spaces. On the other hand, the Besov-Morrey space $\mathcal{N}_{p, q, u}^{\alpha}$ is a proper subspace of the space $B_{u, q}^{\alpha, \frac{1}{q}-\frac{1}{p}}$ with $u<p$ and $q<\infty$ (see [31]). Further properties for these function spaces can be found in [28], [29], and [30].

Recently, Triebel [36] further introduced and studied some local versions of these smoothness Morrey-type spaces and also considered their applications in heat equations and Navier-Stokes equations. More recent results can be found in [44], where they studied the relations between Triebel's local spaces and the Besov-type and Triebel-Lizorkin-type spaces and their associated uniform spaces.

D. Yang and W. Yuan [39], [40] introduced and investigated the homogeneous Besov and Triebel-Lizorkin spaces, which generalize the homogeneous Besov and Triebel-Lizorkin spaces.

The Besov-Morrey spaces with variable exponents were first introduced in [14], which also introduced equivalent quasinorms of these new spaces, which are formulated in terms of Peetre's maximal functions. Also the authors obtained the atomic, molecular, and wavelet decompositions of these new spaces.

In the next proposition we present the relations between variable BesovMorrey spaces and variable Besov-type spaces (see [13]).

\section{PROPOSITION 3.8}

Let $\alpha \in C_{\mathrm{loc}}^{\mathrm{log}}, 0<q<\infty$, and $0<p<u<\infty$.

(i) For $0<q<\infty$ we have the continuous embeddings

$$
\mathcal{N}_{u, q, p}^{\alpha(\cdot)} \hookrightarrow B_{p, q}^{\alpha(\cdot),\left(\frac{1}{p}-\frac{1}{u}\right)^{-1}}
$$

(ii) We have

$$
\mathcal{N}_{u, \infty, p}^{\alpha(\cdot)}=B_{p, \infty}^{\alpha(\cdot),\left(\frac{1}{p}-\frac{1}{u}\right)^{-1}}
$$

Following [9], we define, for $a>0, v \in \mathbb{N}_{0}, \alpha: \mathbb{R}^{n} \rightarrow \mathbb{R}$, and $f \in \mathcal{S}^{\prime}\left(\mathbb{R}^{n}\right)$, the Peetre maximal function

$$
\begin{aligned}
& \varphi_{v}^{*, a} 2^{v \alpha(\cdot)} f(x)=\sup _{y \in \mathbb{R}^{n}} \frac{2^{v \alpha(y)}\left|\varphi_{v} * f(y)\right|}{\left(1+2^{v}|x-y|\right)^{a}} \quad \text { and } \\
& \varphi_{v, P}^{*, a} 2^{v \alpha(\cdot)} f(x)=\sup _{y \in P} \frac{2^{v \alpha(y)}\left|\varphi_{v} * f(y)\right|}{\left(1+2^{v}|x-y|\right)^{a}}
\end{aligned}
$$


where $P$ is a dyadic cube. We now present a fundamental characterization of the spaces under consideration.

\section{THEOREM 3.9}

Let $\alpha \in C_{\operatorname{loc}}^{\log }$ and $p, q, \tau \in \mathcal{P}_{0}^{\log }$. Let $a>\frac{2 n+c_{\log }(1 / \tau)+c_{\log }(1 / q)}{p^{-}}$and $0<q^{-} \leq$ $q^{+}<\infty$. Then

$$
\|f\|_{B_{p(\cdot), q(\cdot)}^{\alpha(\cdot), \tau(\cdot)}}:=\sup _{P \in \mathcal{Q}}\left\|\left(\frac{\varphi_{v}^{*, a} 2^{v \alpha(\cdot)} f}{\left\|\chi_{P}\right\|_{\tau(\cdot)}} \chi_{P}\right)_{v \geq v_{P}^{+}}\right\|_{\ell^{q(\cdot)}\left(L^{p(\cdot)}\right)}
$$

is an equivalent quasinorm in $B_{p(\cdot), q(\cdot)}^{\alpha(\cdot), \tau(\cdot)}$.

Proof

We divide the proof into two steps.

Step 1 . It is easy to see that for any $f \in \mathcal{S}^{\prime}\left(\mathbb{R}^{n}\right)$ with $\|f\|_{B_{p(\cdot), q(\cdot)}^{\alpha(\cdot), \tau(\cdot)}}^{\nabla}<\infty$ and any $x \in \mathbb{R}^{n}$ we have

$$
2^{v \alpha(x)}\left|\varphi_{v} * f(x)\right| \leq \varphi_{v}^{*, a} 2^{v \alpha(\cdot)} f(x) .
$$

This shows that the right-hand side in (3.4) is less than or equal to (3.10).

Step 2. We will prove in this step that there is a constant $C>0$ such that for every $f \in B_{p(\cdot), q(\cdot)}^{\alpha(\cdot), \tau(\cdot)}$

$$
\|f\|_{B_{p(\cdot), q(\cdot)}^{\alpha(\cdot), \tau(\cdot)}}^{\nabla} \leq C\|f\|_{B_{p(\cdot), q(\cdot)}^{\alpha(\cdot), \tau(\cdot)}} .
$$

We choose $t>0$ such that $a>\frac{2 n+c_{\log }(1 / \tau)+c_{\log }(1 / q)}{t}>\frac{2 n+c_{\log }(1 / \tau)+c_{\log }(1 / q)}{p^{-}}$. By Lemmas 2.6 and 2.5 the estimates

$$
\begin{aligned}
2^{v \alpha(y)}\left|\varphi_{v} * f(y)\right| & \leq C_{1} 2^{v \alpha(y)}\left(\eta_{v, m} *\left|\varphi_{v} * f\right|^{t}(y)\right)^{1 / t} \\
& \leq C_{2}\left(\eta_{v, m-c_{\log }(\alpha)} *\left(2^{v \alpha(\cdot)}\left|\varphi_{v} * f\right|\right)^{t}(y)\right)^{1 / t}
\end{aligned}
$$

are true for any $y \in \mathbb{R}^{n}, v \in \mathbb{N}_{0}$, and any $m>0$. Now divide both sides of (3.12) by $\left(1+2^{v}|x-y|\right)^{a}$. On the right-hand side we use the inequality

$$
\left(1+2^{v}|x-y|\right)^{-a} \leq\left(1+2^{v}|x-z|\right)^{-a}\left(1+2^{v}|y-z|\right)^{a}, \quad x, y, z \in \mathbb{R}^{n} .
$$

On the left-hand side take the supremum over $y \in \mathbb{R}^{n}$ and get for all $f \in B_{p(\cdot), q(\cdot)}^{\alpha(\cdot),}$, any $x \in P$, any $v \geq v_{P}^{+}$, and any $m>a t+c_{\log }(\alpha)$

$$
\left(\varphi_{v}^{*, a} 2^{v \alpha(\cdot)} f(x)\right)^{t} \leq C_{2} \eta_{v, a t} *\left(2^{v \alpha(\cdot)}\left|\varphi_{v} * f\right|\right)^{t}(x),
$$

where $C_{2}>0$ is independent of $x, v$, and $f$. An application of Lemma 2.8(i) gives that the left-hand side of (3.11) is bounded by

$$
\begin{gathered}
C \sup _{P \in \mathcal{Q}}\left\|\left(\frac{\eta_{v, a t} *\left(2^{v \alpha(\cdot)}\left|\varphi_{v} * f\right|\right)^{t}}{\left\|\chi_{P}\right\|_{\tau(\cdot)}^{t}} \chi_{P}\right)_{v \geq v_{P}^{+}}\right\|_{\ell^{\frac{q(\cdot)}{t}}}^{1 / t}{ }_{\left(L^{\frac{p(\cdot)}{t}}\right)} \\
\leq C\left\|\left(2^{v \alpha(\cdot)} \varphi_{v} * f\right)_{v}\right\|_{\ell^{\tau(\cdot), q(\cdot)}\left(L^{p(\cdot)}\right)}=C\|f\|_{B_{p(\cdot), q(\cdot)}^{\alpha(\cdot), \tau(\cdot)} .}
\end{gathered}
$$

The proof is complete. 
Notice that, for $a>0, v \in \mathbb{N}_{0}, \alpha: \mathbb{R}^{n} \rightarrow \mathbb{R}, f \in \mathcal{S}^{\prime}\left(\mathbb{R}^{n}\right)$, and $x \in P, 2^{v \alpha(\cdot)} \mid \varphi_{v} *$ $f \mid(x) \leq \varphi_{v, P}^{*, a} 2^{v \alpha(\cdot)} f(x) \leq \varphi_{v}^{*, a} 2^{v \alpha(\cdot)} f(x)$. Therefore, we also have the following maximal function characterizations.

THEOREM 3.13

Let $\alpha \in C_{\operatorname{loc}}^{\log }$ and $p, q, \tau \in \mathcal{P}_{0}^{\log }$. Let $a>\frac{2 n+c_{\log }(1 / \tau)+c_{\log }(1 / q)}{p^{-}}$and $0<q^{-} \leq$ $q^{+}<\infty$. Then

$$
\|f\|_{B_{p(\cdot), q(\cdot)}^{\alpha(\cdot), \tau(\cdot)}}^{*}:=\sup _{P \in \mathcal{Q}}\left\|\left(\frac{\varphi_{v, P}^{*, a} 2^{v \alpha(\cdot)} f}{\left\|\chi_{P}\right\|_{\tau(\cdot)}} \chi_{P}\right)_{v \geq v_{P}^{+}}\right\|_{\ell^{q(\cdot)}\left(L^{p(\cdot)}\right)}
$$

is an equivalent quasinorm in $B_{p(\cdot), q(\cdot)}^{\alpha(\cdot), \tau(\cdot)}$.

The following result is from [12].

LEMMA 3.14

Let $\alpha \in C_{\mathrm{loc}}^{\log }, p, q \in \mathcal{P}_{0}^{\log }$, and $0<q^{+}<\infty$. A tempered distribution $f$ belongs to $\widetilde{B}_{p(\cdot), q(\cdot)}^{\alpha(\cdot), p(\cdot)}$ if and only if

$$
\|f\|_{\widetilde{B}_{p(\cdot), q(\cdot)}^{\alpha(\cdot), p(\cdot)}}^{\#}:=\sup _{\{P \in \mathcal{Q},|P| \leq 1\}}\left\|\left(\frac{2^{v \alpha(\cdot)} \varphi_{v} * f}{|P|^{1 / p(\cdot)}} \chi_{P}\right)_{v \geq v_{P}}\right\|_{\ell^{q(\cdot)}\left(L^{p(\cdot)}\right)}<\infty .
$$

Furthermore, the quasinorms $\|f\|_{\widetilde{B}_{p(\cdot), q(\cdot)}^{\alpha(\cdot), p(\cdot)}}$ and $\|f\|_{\widetilde{B}_{p(\cdot), q(\cdot)}^{\alpha(\cdot), p(\cdot)}}^{\#}$ are equivalent.

Using this lemma, we can easily prove that if $\alpha \in C_{\mathrm{loc}}^{\log }, p, q \in \mathcal{P}_{0}^{\log }$, and $0<q^{+}<$ $\infty$, then $B_{p(\cdot), q(\cdot)}^{\alpha(\cdot),} \hookrightarrow \widetilde{B}_{p(\cdot), q(\cdot)}^{\alpha(\cdot), p(\cdot)}$.

By the same arguments used in the proof of Theorem 3.9, we have the following characterizations of the spaces $\widetilde{B}_{p(\cdot), q(\cdot)}^{\alpha(\cdot), p}$.

THEOREM 3.16

Let $\alpha \in C_{\operatorname{loc}}^{\log }$ and $p, q \in \mathcal{P}_{0}^{\log }$. Let $a>\frac{2 n+c_{\log }(1 / p)+c_{\log }(1 / q)}{p^{-}}$and $0<q^{-} \leq q^{+}<\infty$. Then

$$
\|f\|_{\widetilde{B}_{p(\cdot), q(\cdot)}^{\alpha(\cdot), p(\cdot)}}^{\mathbf{\nabla}}:=\sup _{P \in \mathcal{Q}}\left\|\left(\frac{\varphi_{v}^{*, a} 2^{v \alpha(\cdot)} f}{|P|^{1 / p(\cdot)}} \chi_{P}\right)_{v \geq v_{P}^{+}}\right\|_{\ell q(\cdot)\left(L^{p(\cdot)}\right)}
$$

and

$$
\|f\|_{\widetilde{B}_{p(\cdot), q(\cdot)}^{\alpha(\cdot), p(\cdot)}}:=\sup _{P \in \mathcal{Q}}\left\|\left(\frac{\varphi_{v, P}^{*, a} 2^{v \alpha(\cdot)} f}{|P|^{1 / p(\cdot)}} \chi_{P}\right)_{v \geq v_{P}^{+}}\right\|_{\ell^{q(\cdot)}\left(L^{p(\cdot)}\right)}
$$

are an equivalent quasinorm in $\widetilde{B}_{p(\cdot), q(\cdot)}^{\alpha(\cdot), p(\cdot)}$.

\section{Characterization by ball means of differences}

This section is devoted to the characterization of Besov-type spaces $B_{p(\cdot), q(\cdot)}^{\alpha(\cdot), \tau(\cdot)}$ and $\widetilde{B}_{p(\cdot), q(\cdot)}^{\alpha(\cdot), p(\cdot)}$ by ball means of differences. In the case of constant indices $p, q, \alpha$, and 
$\tau$, we refer especially to [10] and [43]. Let $f$ be an arbitrary function on $\mathbb{R}^{n}$ and $x, h \in \mathbb{R}^{n}$. Then

$$
\Delta_{h} f(x)=f(x+h)-f(x), \quad \Delta_{h}^{M+1} f(x)=\Delta_{h}\left(\Delta_{h}^{M} f\right)(x), \quad M \in \mathbb{N} .
$$

These are the well-known differences of functions which play an important role in the theory of function spaces. Using mathematical induction one can show the explicit formula

$$
\Delta_{h}^{M} f(x):=\sum_{j=0}^{M}(-1)^{j}\left(\begin{array}{c}
M \\
j
\end{array}\right) f(x+(M-j) h),
$$

where $\left(\begin{array}{c}M \\ j\end{array}\right)$ are the binomial coefficients. By ball means of differences we mean the quantity

$$
d_{t}^{M} f(x)=t^{-n} \int_{|h| \leq t}\left|\Delta_{h}^{M} f(x)\right| d h=\int_{B}\left|\Delta_{t h}^{M} f(x)\right| d h,
$$

where $B=\left\{y \in \mathbb{R}^{n}:|h| \leq 1\right\}$ is the unit ball of $\mathbb{R}^{n}, t>0$ is a real number, and $M$ is a natural number. In the following we present some properties of the spaces $L_{\lambda(\cdot)}^{p(\cdot)}$ and $\widetilde{L^{p(\cdot)}}$.

\section{PROPOSITION 4.1}

Let $\tau, \lambda \in \mathcal{P}_{0}^{\log }$ and $p \in \mathcal{P}^{\log }$.

(i) If $\tau_{\infty} \leq \lambda_{\infty}$, then

$$
L_{\lambda(\cdot)}^{p(\cdot)} \hookrightarrow L_{\tau(\cdot)}^{p(\cdot)}
$$

(ii) We have

$$
B_{p(\cdot), 1}^{0, \tau(\cdot)} \hookrightarrow L_{\tau(\cdot)}^{p(\cdot)} \hookrightarrow \widetilde{L^{p(\cdot)}} \hookrightarrow \mathcal{S}^{\prime}\left(\mathbb{R}^{n}\right)
$$

Proof

The property (i) follows from the estimates $\left\|\chi_{P}\right\|_{\lambda(\cdot)} \approx|P|^{1 / \lambda_{\infty}} \leq|P|^{1 / \tau_{\infty}} \approx$ $\left\|\chi_{P}\right\|_{\tau(\cdot)}$ for any $P \in \mathcal{Q}$, with $|P| \geq 1$ (see (2.4)). By Hölder's inequality, we see that for any $\varphi \in \mathcal{S}\left(\mathbb{R}^{n}\right)$ and any $M \in \mathbb{N}$ large enough

$$
\begin{aligned}
|\langle f, \varphi\rangle| & \leq \int_{\mathbb{R}^{n}}|f(x) \| \varphi(x)| d x \\
& =\sum_{m \in \mathbb{Z}^{n}} \int_{Q_{0, m}}|f(x)||\varphi(x)| d x \\
& \leq \sum_{m \in \mathbb{Z}^{n}}\left\|f \chi_{Q_{0, m}}\right\|_{p(\cdot)}\left\|\varphi \chi_{Q_{0, m}}\right\|_{p^{\prime}(\cdot)} \\
& \leq C_{1}\|\varphi\|_{M, 0}\|f\|_{\widetilde{L^{p(\cdot)}}} \sum_{m \in \mathbb{Z}^{n}}(1+|m|)^{-M} \\
& \leq C_{2}\|\varphi\|_{M, 0}\|f\|_{L_{\tau(\cdot)}^{p(\cdot)}}
\end{aligned}
$$


which proves the embedding $L_{\tau(\cdot)}^{p(\cdot)} \hookrightarrow \widetilde{L^{p(\cdot)}} \hookrightarrow \mathcal{S}^{\prime}\left(\mathbb{R}^{n}\right)$. Let $\left\{\mathcal{F} \varphi_{v}\right\}_{v \in \mathbb{N}_{0}}$ be a resolution of unity. Then, for each dyadic cube $P, f=\sum_{v=0}^{\infty} \varphi_{v} * f$ in the sense of $L^{p(\cdot)}(P)$. Thus, if $|P| \geq 1$, then

$$
\left\|\frac{f \chi_{P}}{\left\|\chi_{P}\right\|_{\tau(\cdot)}}\right\|_{p(\cdot)} \leq \sum_{v=0}^{\infty}\left\|\frac{\varphi_{v} * f}{\left\|\chi_{P}\right\|_{\tau(\cdot)}} \chi_{P}\right\|_{p(\cdot)} \leq\|f\|_{B_{p(\cdot), 1}^{0, \tau(\cdot)}}
$$

follows.

Before proving the characterization of these function spaces by ball means of differences we present some technical lemmas.

LEMMA 4.2

Let $R>0, \tau, p \in \mathcal{P}_{0}^{\log }, 0<r<p^{-}$, and $\theta, \omega \in \mathcal{S}\left(\mathbb{R}^{n}\right)$ with supp $\mathcal{F} \omega \subset B(0,1)$.

(i) For any $f \in \mathcal{S}^{\prime}\left(\mathbb{R}^{n}\right)$, any $m>2 n+c_{\log }\left(\frac{1}{\tau}\right) r$, and any dyadic cube $P$ with $|P| \geq 1$, we have

$$
\left\|\frac{\theta_{R} * \omega_{R} * f}{\left\|\chi_{P}\right\|_{\tau(\cdot)}} \chi_{P}\right\|_{p(\cdot)} \leq C \max \left(1,(R l(P))^{(n-m) / r}\right)\left\|\omega_{R} * f\right\|_{L_{\tau(\cdot)}^{p(\cdot)}}
$$

such that the right-hand side is finite, where $C>0$ is independent of $R, f$, and $l(P)$.

(ii) For any $f \in \mathcal{S}^{\prime}\left(\mathbb{R}^{n}\right)$, any $m>2 n$, and any dyadic cube $P$ with $|P|=1$, we have

$$
\left\|\left(\theta_{R} * \omega_{R} * f\right) \chi_{P}\right\|_{p(\cdot)} \leq C \max \left(1, R^{(n-m) / r}\right)\left\|\omega_{R} * f\right\|_{L^{p(\cdot)}}
$$

such that the right-hand side is finite, where $C>0$ is independent of $R$ and $f$.

This lemma is from [12, Lemma 2.4]. Let us now introduce the (quasi-)norms, which shall be the main subject of our study. We define

$$
\|f\|_{B_{p(\cdot), q(\cdot)}^{\alpha(\cdot),(\cdot)}}^{*}:=\|f\|_{L_{\tau(\cdot)}^{p(\cdot)}}+\sup _{P \in \mathcal{Q}}\left\|\left(\frac{2^{k \alpha(\cdot)} d_{2^{-k}}^{M} f}{\left\|\chi_{P}\right\|_{\tau(\cdot)}} \chi_{P}\right)_{k \geq v_{P}^{+}}\right\|_{\ell q(\cdot)\left(L^{p(\cdot)}\right)}
$$

and

$$
\|f\|_{B_{p(\cdot), q(\cdot)}^{\alpha(\cdot), p(\cdot)}}^{*}:=\|f\|_{\widetilde{L^{p(\cdot)}}}+\sup _{\{P \in \mathcal{Q},|P| \leq 1\}}\left\|\left(\frac{2^{k \alpha(\cdot)} d_{2^{-k}}^{M} f}{|P|^{1 / p(\cdot)}} \chi_{P}\right)_{k \geq v_{P}}\right\|_{\ell^{q(\cdot)}\left(L^{p(\cdot)}\right)}
$$

In view of [13, Lemma 3.6], the supremum in (4.3) can be taken with respect to any dyadic cubes. The following technical lemma is just [10, Lemma 3.5] for constant exponents.

\section{LEMMA 4.4}

Let $\alpha \in C_{\mathrm{loc}}^{\mathrm{log}}$, with $\alpha^{-}>0, M \in \mathbb{N}, \tau, q \in \mathcal{P}_{0}^{\log }$, and $p \in \mathcal{P}^{\log }$, with $p^{-}>1$. Then there is a constant $C>0$ such that

$$
\left\|\left(\frac{2^{v \alpha(\cdot)}}{\left\|\chi_{P}\right\|_{\tau(\cdot)}}\left(\int_{|y| \leq 1}\left|\Delta_{2^{-v} y}^{M} f(\cdot)\right| d y\right) \chi_{P}\right)_{v \geq v_{P}^{+}}\right\|_{\ell^{q(\cdot)}\left(L^{p(\cdot)}\right)} \leq C\|f\|_{B_{p(\cdot), q(\cdot)}^{\alpha(\cdot) \tau(\cdot)}}^{*}
$$

and 


$$
\begin{aligned}
& \left\|\left(\frac{2^{v \alpha(\cdot)}}{\left\|\chi_{P}\right\|_{\tau(\cdot)}}\left(\int_{|y|>1}\left|\Delta_{2^{-v} y}^{M} f(\cdot) \omega(y)\right| d y\right) \chi_{P}\right)_{v \geq v_{P}^{+}}\right\|_{\ell^{q(\cdot)}\left(L^{p(\cdot)}\right)} \\
& \quad \leq C\|f\|_{B_{p(\cdot), q(\cdot)}^{\alpha}}^{\alpha(\cdot), \tau(\cdot)}
\end{aligned}
$$

for any dyadic cube $P$, any $\omega \in \mathcal{S}\left(\mathbb{R}^{n}\right)$, and any function $f$ such that $\|f\|_{B_{p(\cdot), q(\cdot)}^{\alpha(\cdot), \tau(\cdot)}}^{*}<\infty$.

Proof

We see that it suffices to prove the second estimate. We write for any $x \in P$

$$
\begin{aligned}
& 2^{v \alpha(x)} \int_{|y|>1}\left|\Delta_{2^{-v} y}^{M} f(x) \omega(y)\right| d y \\
& \quad=\sum_{k=0}^{\infty} 2^{v \alpha(x)} \int_{2^{k}<|y| \leq 2^{k+1}}\left|\Delta_{2^{-v} y}^{M} f(x) \omega(y)\right| d y \\
& \leq C \sum_{k=0}^{\infty} 2^{(\alpha(x)+n) v-N k} \int_{2^{k-v}<|h| \leq 2^{k-v+1}}\left|\Delta_{h}^{M} f(x)\right| d h \\
& =C \sum_{k=0}^{\infty} 2^{-N k} \boldsymbol{\beta}_{v, k}(x),
\end{aligned}
$$

where

$$
\text { q }_{v, k}(x):=2^{(\alpha(x)+n) v} \int_{2^{k-v}<|h| \leq 2^{k-v+1}}\left|\Delta_{h}^{M} f(x)\right| d h,
$$

$N>0$ is at our disposal, and we have used the properties of the function $\omega$, $|\omega(x)| \leq C(1+|x|)^{-N}$ for any $x \in \mathbb{R}^{n}$ and any $N>0$. Fix $0<r<\frac{1}{2} \min \left(p^{-}, q^{-}, 2\right)$. Then the left-hand side of (4.5) is bounded by

$$
\begin{aligned}
& C\left(\sum_{k=0}^{\infty} 2^{-N r k}\left\|\left(\frac{\boldsymbol{\phi}_{v, k}(\cdot)}{\left\|\chi_{P}\right\|_{\tau(\cdot)}} \chi_{P}\right)_{v \geq v_{P}^{+}}\right\|_{\ell^{q(\cdot)}\left(L^{p(\cdot)}\right)}^{r}\right)^{1 / r} \\
& \quad=\left(\sum_{k=0}^{v_{P}^{+}-1} \cdots+\sum_{k=v_{P}^{+}}^{\infty} \cdots\right)^{1 / r} \\
& =\left(I_{v_{P}^{+}}+I I_{v_{P}^{+}}\right)^{1 / r} \\
& \leq 2^{1 / r-1}\left(\left(I_{v_{P}^{+}}\right)^{1 / r}+\left(I I_{v_{P}^{+}}\right)^{1 / r}\right) .
\end{aligned}
$$

Here we put $\sum_{k=0}^{v_{P}^{+}-1} \cdots=0$ if $v_{P}^{+}=0$, and $I_{v_{P}^{+}}$can be rewritten as (here, obviously $\left.v_{P}^{+}=v_{P}\right)$

$$
C \sum_{k=0}^{v_{P}^{+}-2} \cdots+C \sum_{k=v_{P}^{+}-1}^{v_{P}^{+}-1} \cdots=M_{1}+M_{2}
$$


Here we put $\sum_{k=0}^{v_{P}^{+}-2} \cdots=0$ if $v_{P}^{+} \leq 1$. After a change of variable $v-k-1=j$, we get

$$
\begin{aligned}
M_{1} & \leq C \sum_{k=0}^{v_{P}^{+}-2} 2^{\left(\alpha^{+}+n-N\right) r k}\left\|\left(\frac{2^{j \alpha(\cdot)} d_{2^{-j}}^{M} f(\cdot)}{\left\|\chi_{P}\right\|_{\tau(\cdot)}} \chi_{P}\right)_{j \geq v_{P}^{+}-k-1}\right\|_{\ell^{q(\cdot)}\left(L^{p(\cdot)}\right)}^{r} \\
& \leq C \sum_{k=0}^{v_{P}^{+}-2} 2^{\left(\alpha^{+}+n-N\right) r k}\left\|\left(\frac{2^{j \alpha(\cdot)} d_{2^{-j}}^{M} f(\cdot)}{\left\|\chi_{P}\right\|_{\tau(\cdot)}} \chi_{Q\left(x_{P}, 2^{k+1-v_{P}}\right)}\right)_{j \geq v_{P}^{+}-k-1}\right\|_{\ell^{q(\cdot)}\left(L^{p(\cdot)}\right)}^{r},
\end{aligned}
$$

after using $P \subset Q\left(x_{P}, \sqrt{n} 2^{k+1-v_{P}}\right.$ ) (cube centered at the point $x_{P}$ with sides parallel to coordinate axes and of length $\left.\sqrt{n} 2^{k+1-v_{P}}\right)$. This expression is bounded by

$$
C \sum_{k=0}^{v_{P}^{+}-2} 2^{\left(\alpha^{+}+n-N+n / \tau^{-}\right) r k}\left(\|f\|_{B_{p(\cdot), q(\cdot)}^{\alpha(\cdot),(\cdot)}}^{*}\right)^{r} \leq C\left(\|f\|_{B_{p(\cdot), q(\cdot)}^{\alpha(\cdot), \tau(\cdot)}}^{*}\right)^{r},
$$

where we used, by Lemma 2.7,

$$
\frac{\left\|\chi_{Q\left(x_{P}, \sqrt{n} 2^{\left.k+1-v_{P}\right)}\right.}\right\|_{\tau(\cdot)}}{\left\|\chi_{P}\right\|_{\tau(\cdot)}} \leq C 2^{n k / \tau^{-}}
$$

for $N$ large enough such that $N>2\left(\alpha^{+}+n\right)+n / \tau^{-}$and here $C>0$ is independent of $v_{P}$. Now $M_{2}$ is bounded by

$$
C 2^{\left(\alpha^{+}+n-N\right) r v_{P}^{+}}\left\|\left(\frac{2^{v \alpha(\cdot)} d_{2^{-v}}^{M} f(\cdot)}{\left\|\chi_{P}\right\|_{\tau(\cdot)}} \chi_{P}\right)_{v \geq 0}\right\|_{\ell^{q(\cdot)}\left(L^{p(\cdot)}\right)}^{r} \cdot
$$

Using the fact that $\chi_{P} \leq \chi_{Q\left(x_{P}, \sqrt{n}\right)},\left\|\chi_{Q\left(x_{P}, \sqrt{n}\right)}\right\|_{\tau(\cdot)} \approx 1$, and $\left\|\chi_{P}\right\|_{\tau(\cdot)} \geq$ $C 2^{-v_{P} n / \tau^{-}}$, we obtain

$$
M_{2} \leq C 2^{\left(\alpha^{+}+n+n / \tau^{-}-N\right) r v_{P}^{+}}\left(\|f\|_{B_{p(\cdot), q(\cdot)}^{\alpha(\cdot), \tau(\cdot)}}^{*}\right)^{r} \leq C\left(\|f\|_{B_{p(\cdot), q(\cdot)}^{\alpha(\cdot), \tau(\cdot)}}^{*}\right)^{r},
$$

because $N>\alpha^{+}+n+n / \tau^{-}$. Therefore,

$$
\left(I_{v_{P}^{+}}\right)^{1 / r} \leq C\|f\|_{B_{p(\cdot), q(\cdot)}^{\alpha(\cdot) \tau(\cdot)}}^{*}
$$

We estimate $I I_{v_{P}^{+}}$. For all $\left(f_{v}\right)_{v \in \mathbb{N}_{0}} \subset \ell^{q(\cdot)}\left(L^{p(\cdot)}\right)$ we set

$$
\left(f_{v}\right)_{v=v_{P}^{+}}^{k+v_{P}^{+}}=\left(f_{v_{P}^{+}}, \ldots, f_{k+v_{P}^{+}}, 0,0, \ldots\right) .
$$

Since $\|\cdot\|_{\ell^{q(\cdot)}\left(L^{p(\cdot)}\right)}$ is a quasinorm in the $\ell^{q(\cdot)}\left(L^{p(\cdot)}\right)$-spaces we obtain, for any dyadic cube $P, I I_{v_{P}^{+}}$is bounded by

$$
\begin{aligned}
& C \sum_{k=v_{P}^{+}}^{\infty} 2^{-N r k} \|\left(\frac{2^{(\alpha(\cdot)+n) v}}{\left\|\chi_{P}\right\|_{\tau(\cdot)}}\right. \\
& \left.\quad \times\left(\int_{|h| \leq 2^{k-v+1}}\left|\Delta_{h}^{M} f(\cdot)\right| d h\right) \chi_{P}\right)_{v=v_{P}^{+}}^{k+v_{P}^{+}} \|_{\ell^{q(\cdot)}\left(L^{p(\cdot)}\right)}^{r}
\end{aligned}
$$




$$
\begin{aligned}
& +C \sum_{k=v_{P}^{+}}^{\infty} 2^{-N r k} \|\left(\frac{2^{(\alpha(\cdot)+n) v}}{\left\|\chi_{P}\right\|_{\tau(\cdot)}}\right. \\
& \left.\times\left(\int_{|h| \leq 2^{k-v+1}}\left|\Delta_{h}^{M} f(\cdot)\right| d h\right) \chi_{P}\right)_{v \geq k+v_{P}^{+}+1} \|_{\ell^{q(\cdot)}\left(L^{p(\cdot)}\right)}^{r} .
\end{aligned}
$$

Using the embedding $\ell^{q^{-}}\left(L^{p(\cdot)}\right) \hookrightarrow \ell^{q(\cdot)}\left(L^{p(\cdot)}\right)$ we estimate the first term by

$$
\begin{aligned}
& C \sum_{k=v_{P}^{+}}^{\infty} 2^{-N r k}\left(\sum_{v=v_{P}^{+}}^{k+v_{P}^{+}}\left\|\frac{2^{(\alpha(\cdot)+n) v}}{\left\|\chi_{P}\right\|_{\tau(\cdot)}}\left(\int_{|h| \leq 2^{k-v_{P}^{+}+1}}\left|\Delta_{h}^{M} f(\cdot)\right| d h\right) \chi_{P}\right\|_{p(\cdot)}^{q^{-}}\right)^{r / q^{-}} \\
& \quad \leq C \sum_{k=v_{P}^{+}}^{\infty} \frac{2^{\left(2\left(\alpha^{+}+n\right)-N\right) r k} k}{\left\|\chi_{P}\right\|_{\tau(\cdot)}^{r}}\left\|\left(\int_{|h| \leq 2^{k-v_{P}^{+}+1}}\left|\Delta_{h}^{M} f(\cdot)\right| d h\right) \chi_{P}\right\|_{p(\cdot)}^{r} .
\end{aligned}
$$

Recalling the definition of $\Delta_{h}^{M} f$, we obtain

$$
\begin{aligned}
& \int_{|h| \leq 2^{k-v_{P}^{+}+1}}\left|\Delta_{h}^{M} f(x)\right| d h \\
& \leq \sum_{m=0}^{M-1}\left(\begin{array}{c}
M \\
m
\end{array}\right) \int_{|h| \leq 2^{k-v_{P}^{+}+1}}|f(x+(M-m) h)| d h+C 2^{n\left(k-v_{P}^{+}\right)}|f(x)| \\
& =C \sum_{m=0}^{M-1}\left(\begin{array}{c}
M \\
m
\end{array}\right) \int_{|y-x| \leq(M-m) 2^{k-v_{P}^{+}+1}}|f(y)| \chi_{Q\left(x_{P},(M+\sqrt{n}) 2^{\left.k+1-v_{P}\right)}\right.}(y) d y \\
& \quad+C 2^{n\left(k-v_{P}^{+}\right)}|f(x)| \\
& \leq C 2^{n\left(k-v_{P}^{+}\right)}\left(|f(x)|+\mathcal{M}\left(f \chi_{Q\left(x_{P},(M+\sqrt{n}) 2^{\left.k+1-v_{P}\right)}\right.}\right)(x)\right)
\end{aligned}
$$

for any $x \in P$. Hence,

$$
\begin{aligned}
& \left\|\left(\int_{|h| \leq 2^{k-v_{P}^{+}+1}}\left|\Delta_{h}^{M} f(\cdot)\right| d h\right) \chi_{P}\right\|_{L^{p(\cdot)}} \\
& \quad \leq C 2^{n\left(k-v_{P}^{+}\right)}\left(\left\|f \chi_{P}\right\|_{p(\cdot)}+\left\|\mathcal{M}\left(f \chi_{Q\left(x_{P},(M+\sqrt{n}) 2^{\left.k+1-v_{P}\right)}\right.}\right)\right\|_{p(\cdot)}\right) \\
& \quad \leq C 2^{n\left(k-v_{P}^{+}\right)}\left(\left\|f \chi_{P}\right\|_{p(\cdot)}+\left\|f \chi_{Q\left(x_{P},(M+\sqrt{n}) 2^{\left.k+1-v_{P}\right)}\right.}\right\|_{p(\cdot)}\right) \\
& \quad \leq C 2^{n\left(k-v_{P}^{+}\right)}\left\|\chi_{Q\left(x_{P},(M+\sqrt{n}) 2^{\left.k+1-v_{P}\right)}\right.}\right\|_{\tau(\cdot)}\|f\|_{L_{\tau(\cdot)}^{p(\cdot)},}
\end{aligned}
$$

after using the fact that $\mathcal{M}: L^{p(\cdot)} \rightarrow L^{p(\cdot)}$ is bounded. We use (4.6) with $Q\left(x_{P},(M+\sqrt{n}) 2^{k+1-v_{P}}\right)$ in place of $Q\left(x_{P}, \sqrt{n} 2^{k+1-v_{P}}\right)$ to estimate the last expression as

$$
C 2^{\left(n+n / \tau^{-}\right) k}\left\|\chi_{P}\right\|_{\tau(\cdot)}\|f\|_{L_{\tau(\cdot)}^{p(\cdot)}} \leq C 2^{\left(n+n / \tau^{-}\right) k}\left\|\chi_{P}\right\|_{\tau(\cdot)}\|f\|_{B_{p(\cdot), q(\cdot)}^{\alpha(\cdot), \tau(\cdot)}}^{*}
$$

Then the first term in (4.7) is bounded by

$$
C \sum_{k=v_{P}^{+}}^{\infty} 2^{\left(2 \alpha^{+}+3 n+n / \tau^{-}-N\right) r k} k\|f\|_{L_{\tau(\cdot)}^{p(\cdot)}}^{r} \leq C\left(\|f\|_{B_{p(\cdot), q(\cdot)}^{\alpha(\cdot),(\cdot)}}^{*}\right)^{r}
$$


where we use the fact that $N>2 \alpha^{+}+3 n+n / \tau^{-}$. The second term in (4.7) is bounded by

$$
\begin{aligned}
& C \sum_{k=v_{P}^{+}}^{\infty} 2^{\left(\alpha^{+}-N+n\right) r k}\left\|\left(\frac{2^{\alpha(\cdot) v} d_{2^{-v}}^{M} f(\cdot)}{\left\|\chi_{P}\right\|_{\tau(\cdot)}} \chi_{P}\right)_{v \geq v_{P}^{+}}\right\|_{\ell^{q(\cdot)}\left(L^{p(\cdot)}\right)}^{r} \\
& \leq C \sum_{k=v_{P}^{+}}^{\infty} 2^{\left(\alpha^{+}-N+n\right) r k}\left(\|f\|_{B_{p(\cdot), q(\cdot)}^{\alpha}}^{\alpha(\cdot), \tau}\right)^{r} \\
& \leq C\left(\|f\|_{B_{p(\cdot), q(\cdot)}^{\alpha(\cdot), \tau(\cdot)}}^{*}\right)^{r}
\end{aligned}
$$

since $N>\alpha^{+}+n$. This finishes the proof of Lemma 4.4.

\section{LEMMA 4.8}

Let $\alpha \in C_{\operatorname{loc}}^{\log }$, with $\alpha^{-}>0, M \in \mathbb{N}, q \in \mathcal{P}_{0}^{\log }$, and $p \in \mathcal{P}^{\log }$, with $p^{-}>1$. Then there is a constant $C>0$ such that

$$
\left\|\left(\frac{2^{v \alpha(\cdot)}}{|P|^{1 / p(\cdot)}}\left(\int_{|y| \leq 1}\left|\Delta_{2^{-v} y}^{M} f(\cdot)\right| d y\right) \chi_{P}\right)_{v \geq v_{P}}\right\|_{\ell^{q(\cdot)}\left(L^{p(\cdot)}\right)} \leq C\|f\|_{\widetilde{B}_{p(\cdot), q(\cdot)}^{\alpha(\cdot), p(\cdot)}}^{*}
$$

and

$$
\left\|\left(\frac{2^{v \alpha(\cdot)}}{|P|^{1 / p(\cdot)}}\left(\int_{|y|>1}\left|\Delta_{2^{-v} y}^{M} f(\cdot) \omega(y)\right| d y\right) \chi_{P}\right)_{v \geq v_{P}}\right\|_{\ell^{q(\cdot)}\left(L^{p(\cdot)}\right)} \leq C\|f\|_{\widetilde{B}_{p(\cdot), q(\cdot)}^{\alpha(\cdot), p(\cdot)}}^{*}
$$

for any dyadic cube $P$, with $|P| \leq 1$, any $\omega \in \mathcal{S}\left(\mathbb{R}^{n}\right)$, and any function $f$ such that $\|f\|_{\widetilde{B}_{p(\cdot), q(\cdot)}^{\alpha(\cdot), p(\cdot)}}^{*}<\infty$.

\section{Proof}

In view of the proof of Lemma 4.4, we need only to estimate the first term in (4.7). (Observe that $\left\|\chi_{P}\right\|_{p(\cdot)} \approx|P|^{1 / p(x)}, x \in P$, with $|P| \leq 1$ (see (2.3)).) For any dyadic cube with $|P| \leq 1$ and $k \geq v_{P}$

$$
\begin{aligned}
& \left\|\frac{1}{\left\|\chi_{P}\right\|_{p(\cdot)}}\left(\int_{|h| \leq 2^{k-v_{P}+1}}\left|\Delta_{h}^{M} f(\cdot)\right| d h\right) \chi_{P}\right\|_{L^{p(\cdot)}} \\
& \quad \leq C 2^{n\left(k-v_{P}\right)}\left(\left\|\frac{f \chi_{P}}{\left\|\chi_{P}\right\|_{p(\cdot)}}\right\|_{p(\cdot)}+\left\|\frac{f \chi_{Q\left(x_{P}, 2^{\left.k+h_{n}-v_{P}\right)}\right.}}{\left\|\chi_{P}\right\|_{p(\cdot)}}\right\|_{p(\cdot)}\right), \quad h_{n} \in \mathbb{N} .
\end{aligned}
$$

Observe that $Q\left(x_{P}, 2^{k+h_{n}-v_{P}}\right) \subset \bigcup_{i=1}^{3^{n}} Q^{i}=\bigcup_{i=1}^{3^{n}} \bigcup_{l=1}^{2^{\left(k+h_{n}-v\right)_{P}}} Q^{i, l}$, where the $Q^{i}$, $\mathrm{s}$ are dyadic cubes with side length $2^{k+h_{n}-v_{P}}$ and the $Q^{i, l}$, s are dyadic cubes with side length 1 . Therefore,

$$
\begin{aligned}
& \left\|\frac{f \chi_{Q\left(x_{P}, 2^{\left.k+h_{n}-v_{P}\right)}\right.}}{\left\|\chi_{P}\right\|_{p(\cdot)}}\right\|_{p(\cdot)} \\
& \leq C 2^{n v_{P} / p^{-}}\left\|f \chi_{Q\left(x_{P}, 2^{\left.k+h_{n}-v_{P}\right)}\right.}\right\|_{p(\cdot)} \\
& \leq C 3^{n v_{P} / p^{-}} \sum_{i=1}^{2^{n}} \sum_{l=1}^{2^{\left(k+h_{n}-v\right)_{P}}}\left\|f \chi_{Q^{i, l}}\right\|_{p(\cdot)} \leq C 2^{n k}\|f\|_{L^{p(\cdot)}},
\end{aligned}
$$


where $C>0$ is independent of $k$. Now obviously,

$$
\left\|\frac{f \chi_{P}}{\left\|\chi_{P}\right\|_{p(\cdot)}}\right\|_{p(\cdot)} \leq C 2^{n k / p^{-}}\|f\|_{\widetilde{L^{p(\cdot)}}} .
$$

Then the first term in (4.7) is bounded by

$$
C \sum_{k=v_{P}}^{\infty} 2^{\left(2 \alpha^{+}+3 n+n / p^{-}-N\right) r k} k\|f\|_{L^{p(\cdot)}}^{r} \leq C\|f\|_{L^{p(\cdot)}}^{r},
$$

where we use the fact that $N>2 \alpha^{+}+3 n+n / p^{-}$. This finishes the proof of Lemma 4.8.

We set

$$
\delta_{p, \tau}:=n \min \left(0,\left(\frac{1}{p}-\frac{1}{\tau}\right)^{-}\right) .
$$

Using the notation introduced above, we may now state the main result of this article.

\section{THEOREM 4.9}

Let $\alpha \in C_{\mathrm{loc}}^{\mathrm{log}}, M \in \mathbb{N}, \tau, q \in \mathcal{P}_{0}^{\log }$, and $p \in \mathcal{P}^{\log }$, with $p^{-}>1$ and $0<q^{-} \leq$ $q^{+}<\infty$.

(i) Assume

$$
0<\alpha^{-} \leq \alpha^{+}<M+\delta_{p, \tau}
$$

Then $\|\cdot\|_{B_{p(\cdot), q(\cdot)}^{\alpha(\cdot) \tau()}}^{*}$ is an equivalent quasinorm on $B_{p(\cdot), q(\cdot)}^{\alpha(\cdot), \tau(\cdot)}$.

(ii) Assume

$$
0<\alpha^{-} \leq \alpha^{+}<M
$$

Then $\|\cdot\|_{\widetilde{B}_{p(\cdot), q(\cdot)}^{\alpha(\cdot), p}}^{*}$ is an equivalent quasinorm on $\widetilde{B}_{p(\cdot), q(\cdot)}^{\alpha(\cdot), p(\cdot)}$.

Proof

By similarity, we only consider $B_{p(\cdot), q(\cdot)}^{\alpha(\cdot), \tau(\cdot)}$, where for $\widetilde{B}_{p(\cdot), q(\cdot)}^{\alpha(\cdot), p(\cdot)}$ we use the equivalent norm (3.15), Theorem 3.16, and Lemma 4.8 in place of Theorem 3.9 and Lemma 4.4, respectively. Let $P$ be any dyadic cube. We will do the proof in three steps.

Step 1. Let $\left\{\mathcal{F} \varphi_{v}\right\}_{v \in \mathbb{N}_{0}}$ be the functions used in Section 3. We have, with $\alpha^{-}>0$,

$$
\begin{aligned}
\|f\|_{L_{\tau(\cdot)}^{p(\cdot)}} & \leq \sum_{v=0}^{\infty}\left\|\varphi_{v} * f\right\|_{L_{\tau(\cdot)}^{p(\cdot)}} \\
& \leq \sum_{v=0}^{\infty} 2^{-\alpha^{-} v}\left\|2^{\alpha(\cdot) v} \varphi_{v} * f\right\|_{L_{\tau(\cdot)}^{p(\cdot)}} \\
& \leq C\|f\|_{B_{p(\cdot), \infty}^{\alpha(\cdot), \tau(\cdot)}} \leq C\|f\|_{B_{p(\cdot), q(\cdot)}^{\alpha(\cdot), \tau(\cdot)}}
\end{aligned}
$$

for any $f \in B_{p(\cdot), q(\cdot)}^{\alpha(\cdot), \tau(\cdot)}$. 
Step 2. For any $k \geq v_{P}^{+}$and any $x \in P$, we write

$$
\begin{aligned}
\frac{2^{k \alpha(x)}}{\left\|\chi_{P}\right\|_{\tau(\cdot)}} d_{2^{-k}}^{M} f(x) & \leq \sum_{v=0}^{\infty} \frac{2^{k \alpha(x)}}{\left\|\chi_{P}\right\|_{\tau(\cdot)}} d_{2^{-k}}^{M}\left(\varphi_{v} * f\right)(x) \\
& =\sum_{v=0}^{v_{P}^{+}-1} \cdots+\sum_{v=v_{P}^{+}}^{k} \cdots+\sum_{v=k+1}^{\infty} \cdots \\
& =I_{v_{P}}^{k}(x)+I I_{v_{P}}^{k}(x)+I I I_{v_{P}}^{k}(x) .
\end{aligned}
$$

Here we put $I_{v_{P}}^{k}=0$ if $v_{P}^{+}=0$. We estimate the first summand and we start with estimates of the higher-order differences $\Delta_{h}^{M}\left(\varphi_{v} * f\right)$. Let $\psi, \psi_{0} \in \mathcal{S}\left(\mathbb{R}^{n}\right)$ be two functions such that $\mathcal{F} \psi=1$ and $\mathcal{F} \psi_{0}=1$ on $\operatorname{supp} \varphi$ and $\operatorname{supp} \Psi$, respectively. Using the mean value theorem we obtain for any $x \in P, v \in \mathbb{N}_{0}$, and $|h| \leq 2^{-k}$ that

$$
\begin{aligned}
\left|\Delta_{h}^{1}\left(\varphi_{v} * f\right)(x)\right| & =\left|\Delta_{h}^{1}\left(\psi_{v} * \varphi_{v} * f\right)(x)\right| \\
& \leq 2^{-k} \sup _{|x-y| \leq d 2^{-k}} \sum_{|\alpha|=1}\left|D^{\alpha} \psi_{v} * \varphi_{v} * f(y)\right|,
\end{aligned}
$$

with some positive constant $d$, independent of $v$ and $k$, and $\psi_{v}(\cdot)=2^{(v-1) n} \times$ $\psi\left(2^{v-1}\right)$ for $v=1,2, \ldots$ By induction on $M$, we show that

$$
\left|\Delta_{h}^{M}\left(\varphi_{v} * f\right)(x)\right| \leq C 2^{(v-k) M} \sup _{|x-y| \leq d 2^{-k}} \eta_{v-1, N} *\left|\varphi_{v} * f\right|(y),
$$

with $N>0$ large enough. We use Lemma 2.5 to obtain for any $y \in B\left(x, d 2^{-k}\right)$ and any $k \geq v$

$$
\begin{aligned}
& 2^{v(\alpha(x)+n(1 / \tau(x)-1 / p(x)))} \\
& \quad \leq C\left(1+2^{v}|x-y|\right)^{c_{\log }(\alpha+n(1 / \tau-1 / p))} 2^{v(\alpha(y)+n(1 / \tau(y)-1 / p(y)))} \\
& \quad \leq C\left(1+d 2^{v-k}\right)^{c_{\log }(\alpha+n(1 / \tau-1 / p))} 2^{v(\alpha(y)+n(1 / \tau(y)-1 / p(y)))} \\
& \quad \leq C 2^{v(\alpha(y)+n(1 / \tau(y)-1 / p(y)))}
\end{aligned}
$$

with some positive constant $C$ that is independent of $v$. Therefore,

$$
\begin{aligned}
& 2^{v(\alpha(x)+n(1 / \tau(x)-1 / p(x)))}\left|\Delta_{h}^{M}\left(\varphi_{v} * f\right)(x)\right| \\
& \leq C 2^{(v-k) M} \sup _{|x-y| \leq d 2^{-k}} 2^{v(\alpha(y)+n(1 / \tau(y)-1 / p(y)))} \eta_{v-1, N} *\left|\varphi_{v} * f\right|(y) \\
& \leq C 2^{(v-k) M} \sup _{|x-y| \leq d 2^{-k}} \eta_{v-1, N_{1}} * 2^{v(\alpha(\cdot)+n(1 / \tau(\cdot)-1 / p(\cdot)))}\left|\varphi_{v} * f\right|(y),
\end{aligned}
$$

again by Lemma 2.5, with $N_{1}>0$ large enough. The right-hand side may be estimated as

$$
C 2^{(v-k) M} \sup _{|x-y| \leq d 2^{-k}} \varphi_{v}^{*, a} 2^{v(\alpha(\cdot)+n(1 / \tau(\cdot)-1 / p(\cdot)))} f(y) .
$$


Then we obtain, for any $x \in P,|h| \leq 2^{-k}$, and any $k \geq v$, that

$$
2^{v(\alpha(x)+n(1 / \tau(x)-1 / p(x)))}\left|\Delta_{h}^{M}\left(\varphi_{v} * f\right)(x)\right|
$$

can be estimated by

$$
\begin{aligned}
& C 2^{(v-k) M} \sup _{|x-y| \leq d 2^{-k}} \frac{\varphi_{v}^{*, a} 2^{v(\alpha(\cdot)+n(1 / \tau(\cdot)-1 / p(\cdot)))} f(y)}{\left(1+2^{v}|x-y|\right)^{a}} \\
& \leq C 2^{(v-k) M} \varphi_{v}^{*, a} 2^{v(\alpha(\cdot)+n(1 / \tau(\cdot)-1 / p(\cdot)))} f(x) \leq C 2^{(v-k) M}\|f\|_{B_{p(\cdot), q(\cdot)}^{\alpha(\cdot),(\cdot)}}
\end{aligned}
$$

by (3.5). Then

$$
\begin{aligned}
I_{v_{P}}^{k}(x) & \leq C \sum_{v=0}^{v_{P}^{+}} \frac{2^{(v-k)(M-\alpha(x))+v(n / p(x)-n / \tau(x))}}{\left\|\chi_{P}\right\|_{\tau(\cdot)}}\|f\|_{B_{p(\cdot), q(\cdot)}^{\alpha(\cdot), \tau(\cdot)}} \\
& \leq C 2^{k(\alpha(x)-M)} \sum_{v=0}^{v_{P}^{+}} \frac{2^{v(M+n / p(x)-\alpha(x)-n / \tau(x))}}{\left\|\chi_{P}\right\|_{\tau(\cdot)}}\|f\|_{B_{p(\cdot), q(\cdot)}^{\alpha(\cdot)}} \\
& \leq C 2^{k(\alpha(x)-M)} \frac{2^{v_{P}^{+}(M+n / p(x)-\alpha(x)-n / \tau(x))}}{\left\|\chi_{P}\right\|_{\tau(\cdot)}}\|f\|_{B_{p(\cdot), q(\cdot)}^{\alpha(\cdot), \tau(\cdot)}} .
\end{aligned}
$$

Therefore,

$$
I_{v_{P}}^{k}(x) \leq C 2^{v_{P}^{+} n / p(x)} 2^{\left(v_{P}^{+}-k\right)(M-\alpha(x))}\|f\|_{B_{p(\cdot), q(\cdot)}^{\alpha(\cdot), \tau(\cdot)}}, \quad x \in P,
$$

where we have used the fact that $\left\|\chi_{P}\right\|_{\tau(\cdot)} \approx 2^{-v_{P}^{+} n / \tau(x)}$ since $v_{P}^{+}>0$. Taking the $\ell^{q(\cdot)}\left(L^{p(\cdot)}\right)$-(quasi-)norm we obtain

$$
\begin{aligned}
& \sup _{P \in \mathcal{Q}}\left\|\left(I_{v_{P}}^{k} \chi_{P}\right)_{k \geq v_{P}^{+}}\right\|_{\ell^{q(\cdot)}\left(L^{p(\cdot)}\right)} \\
& \leq C\|f\|_{B_{p(\cdot), q(\cdot)}^{\alpha(\cdot),(\cdot)}} \sup _{P \in \mathcal{Q}}\left\|\left(2^{v_{P}^{+} n / p(\cdot)} 2^{\left(v_{P}^{+}-k\right)(M-\alpha(\cdot))} \chi_{P}\right)_{k \geq v_{P}^{+}}\right\|_{\ell^{q(\cdot)}\left(L^{p(\cdot)}\right)} .
\end{aligned}
$$

Let us prove that for any dyadic cube $P$

$$
\left\|\left(2^{v_{P}^{+} n / p(\cdot)} 2^{\left(v_{P}^{+}-k\right)(M-\alpha(\cdot))} \chi_{P}\right)_{k \geq v_{P}^{+}}\right\|_{\ell^{q(\cdot)}\left(L^{p(\cdot)}\right)} \leq C .
$$

By using the embedding $\ell^{q^{-}}\left(L^{p(\cdot)}\right) \hookrightarrow \ell^{q(\cdot)}\left(L^{p(\cdot)}\right)$, the left-hand side is bounded by

$$
C\left(\sum_{k=v_{P}^{+}}^{\infty} 2^{\left(v_{P}^{+}-k\right)\left(M-\alpha^{+}\right) q^{-}}\right)^{1 / q^{-}}\left\|2^{v_{P}^{+} n / p(\cdot)} \chi_{P}\right\|_{p(\cdot)} \leq C\left\|2^{v_{P}^{+} n / p(\cdot)} \chi_{P}\right\|_{p(\cdot)} \leq C,
$$

where we used the fact that $\alpha^{+}<M$ and $\left\|2^{v_{P}^{+} n / p(\cdot)} \chi_{P}\right\|_{p(\cdot)} \leq C$. The second term $I I_{v_{P}}^{k}(x)$ is bounded by

$$
C \sum_{v=v_{P}^{+}}^{k} \frac{2^{(v-k)\left(M-\alpha^{+}\right)}}{\left\|\chi_{P}\right\|_{\tau(\cdot)}} \varphi_{v}^{*, a} 2^{v \alpha(\cdot)} f(x)
$$


where we choose $a>\frac{2 n+c_{\log }(1 / \tau)+c_{\log }(1 / q)}{p^{-}}$. Applying Lemma 2.10, we have

$$
\begin{aligned}
& \sup _{P \in \mathcal{Q}}\left\|\left(I I_{v_{P}}^{k} \chi_{P}\right)_{k \geq v_{P}^{+}}\right\|_{\ell^{q(\cdot)}\left(L^{p(\cdot)}\right)} \\
& \quad \leq C \sup _{P \in \mathcal{Q}}\left\|\left(\frac{\varphi_{v}^{*, a} 2^{v \alpha(\cdot)} f}{\left\|\chi_{P}\right\|_{\tau(\cdot)}} \chi_{P}\right)_{v \geq v_{P}^{+}}\right\|_{\ell^{q(\cdot)}\left(L^{p(\cdot)}\right)} \\
& \quad \leq C\|f\|_{B_{p(\cdot), q(\cdot)}^{\alpha(\cdot), \tau(\cdot)}}
\end{aligned}
$$

where we have used Theorem 3.9. Recalling the definition of $d_{2^{-k}}^{M}\left(\varphi_{v} * f\right)$, we have

$$
\begin{aligned}
& 2^{v \alpha(x)} d_{2^{-k}}^{M}\left(\varphi_{v} * f\right)(x) \\
& \quad=\int_{B} 2^{v \alpha(x)}\left|\Delta_{2^{-k} h}^{M}\left(\varphi_{v} * f\right)(x)\right| d h \\
& \quad \leq \sum_{j=0}^{M}\left(\begin{array}{c}
M \\
j
\end{array}\right) \int_{B} 2^{v \alpha(x)}\left|\left(\varphi_{v} * f\right)\left(x+(M-j) 2^{-k} h\right)\right| d h .
\end{aligned}
$$

We shall deal in detail only with the term with $j=0,1, \ldots, M-1$. The term with $j=M$ is much simpler to handle. (For that term, the integration over $h \in B$ immediately disappears.) We use Lemma 2.6 with $r=1$ in the form $2^{v \alpha(x)}\left|\left(\varphi_{v} * f\right)\left(x+(M-j) 2^{-k} h\right)\right| \leq C 2^{v \alpha(x)} \eta_{v, 2 m} *\left|\varphi_{v} * f\right|\left(x+(M-j) 2^{-k} h\right)$.

We have

$$
\begin{aligned}
& 2^{v \alpha(x)} \int_{B} \eta_{v, 2 m} *\left|\varphi_{v} * f\right|\left(x+(M-j) 2^{-k} h\right) d h \\
& \quad=(M-j)^{-n} 2^{v \alpha(x)+k n} \int_{(M-j) 2^{-k} B} \eta_{v, 2 m} *\left|\varphi_{v} * f\right|(x-t) d t \\
& \quad \leq C 2^{v \alpha(x)} \int_{\mathbb{R}^{n}} \eta_{v, 2 m} *\left|\varphi_{v} * f\right|(x-t) \eta_{k, 2 m}(t) d t \\
& \quad=C 2^{v \alpha(x)} \eta_{k, 2 m} * \eta_{v, 2 m} *\left|\varphi_{v} * f\right|(x) \\
& \quad \leq C 2^{v \alpha(x)} \eta_{k, 2 m} *\left|\varphi_{v} * f\right|(x) \\
& \quad \leq C \eta_{k, m} *\left|2^{v \alpha(\cdot)} \varphi_{v} * f\right|(x),
\end{aligned}
$$

where we have used Lemmas 2.9 and 2.5 with $m>\max \left(n, c_{\log }(\alpha)\right)$. Hence,

$$
2^{v \alpha(x)} d_{2^{-k}}^{M}\left(\varphi_{v} * f\right)(x) \leq C \eta_{k, m} *\left|2^{v \alpha(\cdot)} \varphi_{v} * f\right|(x)
$$

and

$$
\begin{aligned}
I I I_{v_{P}}^{k}(x) & \leq C \sum_{v=k}^{\infty} \frac{2^{(k-v) \alpha(x)}}{\left\|\chi_{P}\right\|_{\tau(\cdot)}} \eta_{k, m} *\left|2^{v \alpha(\cdot)} \varphi_{v} * f\right|(x) \\
& =C \sum_{i=0}^{\infty} \frac{2^{-i \alpha(x)}}{\left\|\chi_{P}\right\|_{\tau(\cdot)}} \eta_{k, m} *\left|2^{(k+i) \alpha(\cdot)} \varphi_{k+i} * f\right|(x)
\end{aligned}
$$


Take the $\ell^{q(\cdot)}\left(L^{p(\cdot)}\right)$-(quasi-)norm, and use Lemma 2.8(i) to obtain (with $m$ large enough and $\left.0<2 r<\min \left(p^{-}, q^{-}, 2\right)\right)$

$$
\begin{aligned}
& \sup _{P \in \mathcal{Q}}\left\|\left(I I I_{v_{P}}^{k} \chi_{P}\right)_{k \geq v_{P}^{+}}\right\|_{\ell^{q(\cdot)}\left(L^{p(\cdot)}\right)}^{r} \\
& \quad \leq C \sum_{i=0}^{\infty} 2^{-i r \alpha^{-}} \sup _{P \in \mathcal{Q}}\left\|\left(\frac{\eta_{k, m} *\left|2^{(k+i) \alpha(\cdot)} \varphi_{k+i} * f\right|}{\left\|\chi_{P}\right\|_{\tau(\cdot)}} \chi_{P}\right)_{k \geq v_{P}^{+}}\right\|_{\ell^{q(\cdot)}\left(L^{p(\cdot)}\right)}^{r} \\
& \quad \leq C \sup _{P \in \mathcal{Q}}\left\|\left(\frac{2^{v \alpha(\cdot)} \varphi_{v} * f}{\left\|\chi_{P}\right\|_{\tau(\cdot)}} \chi_{P}\right)_{v \geq v_{P}^{+}}\right\|_{\ell q(\cdot)\left(L^{p(\cdot))}\right.}^{r}=C\|f\|_{B_{p(\cdot), q(\cdot)}^{\alpha(\cdot),(\cdot)}}^{r} .
\end{aligned}
$$

Step 3. Let $\Psi$ be the function introduced in Section 3 that is, in addition, radially symmetric. We make use of an observation made by Nikol'skij [24] (see also [32] and [35, Section 3.3.2]). We put

$$
\psi(x):=(-1)^{M+1} \sum_{i=0}^{M-1}(-1)^{i}\left(\begin{array}{c}
M \\
i
\end{array}\right) \Psi(x(M-i)) .
$$

The function $\psi$ satisfies $\psi(x)=1$ for $|x| \leq 1 / M$ and $\psi(x)=0$ for $|x| \geq 3 / 2$. Then, taking $\varphi_{0}(x)=\psi(x), \varphi_{1}(x)=\psi(x / 2)-\psi(x)$, and $\varphi_{v}(x)=\varphi_{1}\left(2^{-v+1} x\right)$ for $v=2,3, \ldots$, we obtain that $\left\{\varphi_{v}\right\}$ is a smooth dyadic resolution of unity. This yields that

$$
\sup _{P \in \mathcal{Q}}\left\|\left(\frac{2^{v \alpha(\cdot)} \mathcal{F}^{-1} \varphi_{v} * f}{\left\|\chi_{P}\right\|_{\tau(\cdot)}} \chi_{P}\right)_{v \geq v_{P}^{+}}\right\|_{\ell^{q(\cdot)}\left(L^{p(\cdot)}\right)}
$$

is a quasinorm equivalent in $B_{p(\cdot), q(\cdot)}^{\alpha(\cdot), \tau(\cdot)}$. Let us prove that

$$
\left\|\left(\frac{2^{v \alpha(\cdot)} \mathcal{F}^{-1} \varphi_{v} * f}{\left\|\chi_{P}\right\|_{\tau(\cdot)}} \chi_{P}\right)_{v \geq v_{P}^{+}}\right\|_{\ell^{q(\cdot)}\left(L^{p(\cdot)}\right)} \leq C\|f\|_{B_{p(\cdot), q(\cdot)}^{\alpha(\cdot), \tau(\cdot)}}^{*}
$$

for any dyadic cube $P$ of $\mathbb{R}^{n}$. First, the left-hand side contains $\mathcal{F}^{-1} \varphi_{0} * f$ only when $|P| \geq 1$. Then from Lemma 4.2

$$
\left\|\frac{\mathcal{F}^{-1} \varphi_{0} * f}{\left\|\chi_{P}\right\|_{\tau(\cdot)}} \chi_{P}\right\|_{p(\cdot)} \leq C\|f\|_{B_{p(\cdot), q(\cdot)}^{\alpha(\cdot), \tau(\cdot)}}^{*} .
$$

Moreover, it holds for $x \in \mathbb{R}^{n}$ and $v=1,2, \ldots$ that

$$
\mathcal{F}^{-1} \varphi_{v} * f(x):=(-1)^{M+1} \int_{\mathbb{R}^{n}} \Delta_{2^{-v} y}^{M} f(x) \widetilde{\Psi}(y) d y,
$$

with $\widetilde{\Psi}(\cdot)=\mathcal{F}^{-1} \Psi(\cdot)-2^{-n} \mathcal{F}^{-1} \Psi(\cdot / 2)$ (see [10, Theorem 3.1]). Now, for $v \in \mathbb{N}$ we write

$$
\begin{aligned}
\int_{\mathbb{R}^{n}}\left|\Delta_{2^{-v} y}^{M} f(x)\right||\widetilde{\Psi}(y)| d y= & \int_{|y| \leq 1}\left|\Delta_{2^{-v_{y}}}^{M} f(x)\right||\widetilde{\Psi}(y)| d y \\
& +\int_{|y|>1}\left|\Delta_{2^{-v} y}^{M} f(x)\right||\widetilde{\Psi}(y)| d y
\end{aligned}
$$


Then the estimate (4.10) is an obvious consequence of (4.11) and Lemma 4.4. Therefore, $\|f\|_{B_{p(\cdot), q(\cdot)}^{\alpha(\cdot), \tau(\cdot)}} \leq C\|f\|_{B_{p(\cdot), q(\cdot)}^{\alpha(\cdot),(\cdot)}}^{*}$, which completes the proof of this theorem.

Acknowledgments. We thank the anonymous referee for pointing out the references [7], [19], [20], [23], [31], [36], [39]-[41], [44], [37], and [38] to us and for the valuable comments and suggestions, especially the motivation for introducing the fourth parameter $\tau$.

\section{References}

[1] A. Almeida and P. Hästö, Besov spaces with variable smoothness and integrability, J. Funct. Anal. 258 (2010), 1628-1655. MR 2566313. DOI 10.1016/j.jfa.2009.09.012.

[2] Y. Chen, S. Levine, and R. Rao, Variable exponent, linear growth functionals in image restoration, SIAM J. Appl. Math. 66 (2006), 1383-1406. MR 2246061. DOI 10.1137/050624522.

[3] D. Cruz-Uribe, A. Fiorenza, J. M. Martell, and C. Pérez, The boundedness of classical operators on variable $L^{p}$ spaces, Ann. Acad. Sci. Fenn. Math. 31 (2006), 239-264. MR 2210118.

[4] L. Diening, Maximal function on generalized Lebesque spaces $L^{p(\cdot)}$, Math. Inequal. Appl. 7 (2004), 245-253. MR 2057643. DOI 10.7153/mia-07-27.

[5] L. Diening, P. Hästö, and S. Roudenko, Function spaces of variable smoothness and integrability, J. Funct. Anal. 256 (2009), 1731-1768. MR 2498558. DOI 10.1016/j.jfa.2009.01.017.

[6] L. Diening, P. Harjulehto, P. Hästö, Y. Mizuta, and T. Shimomura, Maximal functions in variable exponent spaces: limiting cases of the exponent, Ann. Acad. Sci. Fenn. Math. 34 (2009), 503-522. MR 2553809.

[7] L. Diening, P. Harjulehto, P. Hästö, and M. Růžička, Lebesgue and Sobolev Spaces with Variable Exponents, Lecture Notes in Math. 2017, Springer, Heidelberg, 2011. MR 2790542. DOI 10.1007/978-3-642-18363-8.

[8] D. Drihem, Some embeddings and equivalent norms of the $\mathcal{L}_{p, q}^{\lambda, s}$ spaces, Funct. Approx. Comment. Math. 41 (2009), 15-40. MR 2568794. DOI $10.7169 / \mathrm{facm} / 1254330157$.

[9] - Atomic decomposition of Besov spaces with variable smoothness and integrability, J. Math. Anal. Appl. 389 (2012), 15-31. MR 2876477. DOI 10.1016/j.jmaa.2011.11.035.

[10] Characterizations of Besov-type and Triebel-Lizorkin-type spaces by differences, J. Funct. Spaces Appl. 2012, no. 328908. MR 2873709. DOI $10.1155 / 2012 / 328908$.

[11] Atomic decomposition of Besov-type and Triebel-Lizorkin-type spaces, Sci. China Math. 56 (2013), 1073-1086. MR 3047054. DOI 10.1007/s11425-012-4425-8. 
[12] Some characterizations of variable Besov-type spaces, Ann. Funct. Anal. 6 (2015), 255-288. MR 3365996. DOI 10.15352/afa/06-4-255.

[13] Some properties of variable Besov-type spaces, Funct. Approx. Comment. Math. 52 (2015), 193-221. MR 3358316.

DOI 10.7169/facm/2015.52.2.2.

[14] J. Fu and J. Xu, Characterizations of Morrey type Besov and Triebel-Lizorkin spaces with variable exponents, J. Math. Anal. Appl. 381 (2011), 280-298. MR 2796209. DOI 10.1016/j.jmaa.2011.02.026.

[15] P. Harjulehto, P. Hästö, Ú. V. Lê, and M. Nuortio, Overview of differential equations with non-standard growth, Nonlinear Anal. 72 (2010), 4551-4574. MR 2639204. DOI 10.1016/j.na.2010.02.033.

[16] H. Kempka and J. Vybíral, Spaces of variable smoothness and integrability: characterizations by local means and ball means of differences, J. Fourier Anal. Appl. 18 (2012), 852-891. MR 2984372. DOI 10.1007/s00041-012-9224-7.

[17] A note on the spaces of variable integrability and summability of Almeida and Hästö, Proc. Amer. Math. Soc. 141 (2013), 3207-3212. MR 3068973. DOI 10.1090/S0002-9939-2013-11605-9.

[18] O. Kováčik and J. Rákosník, On spaces $L^{p(x)}$ and $W^{k, p(x)}$, Czechoslovak Math. J. 41 (1991), 592-618. MR 1134951.

[19] H. Kozono and M. Yamazaki, Semilinear heat equations and the Navier-Stokes equation with distributions in new function spaces as initial data, Comm. Partial Differential Equations 19 (1994), 959-1014. MR 1274547. DOI 10.1080/03605309408821042.

[20] Y. Liang, D. Yang, W. Yuan, Y. Sawano, and T. Ullrich, A new framework for generalized Besov-type and Triebel-Lizorkin-type spaces, Dissertationes Math. (Rozprawy Mat.) 489 (2013), 1-114. MR 3099066. DOI 10.4064/dm489-0-1.

[21] A. L. Mazzucato, Besov-Morrey spaces: function space theory and applications to non-linear PDE, Trans. Amer. Math. Soc. 355 (2003), no. 4, 1297-1364. MR 1946395. DOI 10.1090/S0002-9947-02-03214-2.

[22] C. B. Morrey, Jr., On the solutions of quasi-linear elliptic partial differential equations, Trans. Amer. Math. Soc. 43 (1938), no. 1, 126-166. MR 1501936. DOI $10.2307 / 1989904$.

[23] Y. V. Netrusov, Some imbedding theorems for spaces of Besov-Morrey type (in Russian), Zap. Nauchn. Sem. Leningrad. Otdel. Mat. Inst. Steklov. (LOMI) 139 (1984), 139-147. MR 0756651.

[24] S. M. Nikol'skiŭ, Approximation of Functions of Several Variables and Imbedding Theorems, Grundlehren Math. Wiss. 205, Springer, New York, 1975. MR 0374877.

[25] J. Peetre, On the theory of $\mathcal{L}_{p, \lambda}$ spaces, J. Funct. Anal. 4 (1969), 71-87. MR 0241965.

[26] M. Růžička, Electrorheological Fluids: Modeling and Mathematical Theory, Lecture Notes in Math. 1748, Springer, Berlin, 2000. MR 1810360.

DOI 10.1007/BFb0104029. 
[27] I. I. Šarapudinov, The topology of the space $L^{p(t)}([0,1])$ (in Russian), Mat. Zametki 26 (1979), no. 4, 613-632, 655. MR 0552723.

[28] Y. Sawano, Wavelet characterizations of Besov-Morrey and Triebel-Lizorkin-Morrey spaces, Funct. Approx. Comment. Math. 38 (2008), 93-107. MR 2433791. DOI 10.7169/facm/1229624654.

[29] - A note on Besov-Morrey spaces and Triebel-Lizorkin-Morrey spaces, Acta Math. Sin. (Engl. Ser.) 25 (2009), 1223-1242. MR 2524944.

DOI 10.1007/s10114-009-8247-8.

[30] _ Brezis-Gallouët-Wainger type inequality for Besov-Morrey spaces, Studia Math. 196 (2010), 91-101. MR 2564483. DOI 10.4064/sm196-1-6.

[31] Y. Sawano, D. Yang, and W. Yuan, New applications of Besov-type and Triebel-Lizorkin-type spaces, J. Math. Anal. Appl. 363 (2010), 73-85. MR 2559042. DOI 10.1016/j.jmaa.2009.08.002.

[32] W. Sickel, On pointwise multipliers for $F_{p, q}^{s}\left(\mathbb{R}^{n}\right)$ in case $\sigma_{p, q}<s<n / p$, Ann. Mat. Pura Appl. (4) 176 (1999), 209-250. MR 1746543.

DOI $10.1007 / B F 02505997$.

[33] J.-O. Strömberg and A. Torchinsky, Weighted Hardy Spaces, Lecture Notes in Math. 1381, Springer, Berlin, 1989. MR 1011673.

[34] H. Triebel, Theory of Function Spaces, Monogr. Math. 78, Birkhäuser, Basel, 1983. MR 0781540. DOI 10.1007/978-3-0346-0416-1.

[35] , Theory of Function Spaces, II, Monogr. Math. 84, Birkhäuser, Basel, 1992. MR 1163193. DOI 10.1007/978-3-0346-0419-2.

[36] Local Function Spaces, Heat and Navier-Stokes Equations, EMS Tracts in Math. 20, Eur. Math. Soc. (EMS), Zürich, 2013. MR 3086433. DOI $10.4171 / 123$.

[37] J. Xu, Variable Besov and Triebel-Lizorkin spaces, Ann. Acad. Sci. Fenn. Math. 33 (2008), 511-522. MR 2431378.

[38] _ An atomic decomposition of variable Besov and Triebel-Lizorkin spaces, Armen. J. Math. 2 (2009), 1-12. MR 2485872.

[39] D. Yang and W. Yuan, A new class of function spaces connecting Triebel-Lizorkin spaces and Q spaces, J. Funct. Anal. 255 (2008), 2760-2809. MR 2464191. DOI 10.1016/j.jfa.2008.09.005.

[40] - New Besov-type spaces and Triebel-Lizorkin-type spaces including $Q$ spaces, Math. Z. 265 (2010), 451-480. MR 2609320.

DOI 10.1007/s00209-009-0524-9.

[41] , Relations among Besov-type spaces, Triebel-Lizorkin-type spaces and generalized Carleson measure spaces, Appl. Anal. 92 (2013), 549-561. MR 3021276. DOI 10.1080/00036811.2011.629610.

[42] D. Yang, C. Zhuo, and W. Yuan, Besov-type spaces with variable smoothness and integrability, J. Funct. Anal. 269 (2015), 1840-1898. MR 3373435. DOI 10.1016/j.jfa.2015.05.016. 
[43] W. Yuan, W. Sickel, and D. Yang, Morrey and Campanato Meet Besov, Lizorkin and Triebel, Lecture Notes in Math. 2005, Springer, Berlin, 2010. MR 2683024. DOI 10.1007/978-3-642-14606-0.

[44] On the coincidence of certain approaches to smoothness spaces related to Morrey spaces, Math. Nachr. 286 (2013), 1571-1584. MR 3119702.

[45] W. Yuan, D. D. Haroske, L. Skrzypczak, and D. Yang, Embedding properties of Besov-type spaces, Appl. Anal. 94 (2015), 319-341. MR 3295700. DOI 10.1080/00036811.2014.895331.

Laboratory of Functional Analysis and Geometry of Spaces, Department of Mathematics, M'Sila University, M'Sila, Algeria; douadidr@yahoo.fr 